\title{
THE SYMMETRY AND OPTICAL PHENOMENA
}

\author{
S. MaLinowski \\ Institute of Physics, University of Eódź, Nowotki 149/153, 90-236 Łódź, Poland
}

(Received June 18, 1990)

\begin{abstract}
The explicit forms of the broken (by a electric field $\boldsymbol{E}$, magnetic field $\boldsymbol{H}$ and spatial dispersion of wave vector $k$ ) point symmetry groups of a crystal are given. For these groups the dielectric permeability tensors: $\varepsilon_{i j}(\omega, E)$, $\varepsilon_{i j}(\omega, \boldsymbol{H})$ and $\varepsilon_{i j}(\omega, \boldsymbol{k})$ - the particular cases of $\varepsilon_{i j}(\omega, \boldsymbol{E}, \boldsymbol{H}, \boldsymbol{k})$ - are written out (the susceptibility tensors $\chi_{i j}(\omega, E), \chi_{i j}(\omega, H)$ and $\chi_{i j}(\omega, k)$ take the same forms). In order to illustrate the results obtained the electrooptical phenomena (connected with the tensors $\varepsilon_{i j}(\omega, E)$ ) are discussed.
\end{abstract}

PACS numbers: $75.30 . \mathrm{Cr}, 78.20 . \mathrm{Bh}, 78.20 . \mathrm{Jq}_{\mathrm{q}}$

\section{Introduction}

The electrooptical phenomena obey the same group-theoretical rules as the previously discussed magnetooptic ones [1] and as the phenomena related to the spatial dispersion of the wave vector $k[2,3]$.

The aim of this paper is to present the complete results allowing us to describe the electrooptic phenomena (the optical properties of medium in the external electric field $E$ ), the magnetooptic ones (the optical properties of medium in the external magnetic field $\boldsymbol{H}$ ) as well as the optical properties of the medium in which there is a spontaneous dispersion of wave vector $k$. In Table I the broken symmetry groups $\mathrm{K}(\boldsymbol{F})$ for fields $\boldsymbol{F}=\boldsymbol{E}, \boldsymbol{H}, \boldsymbol{k}$ are given for all point groups $\mathrm{K}$ and all orientations of the fields $\boldsymbol{F}$ with respect to the symmetry elements of the crystals. In Table II the explicit forms of the groups $\mathrm{K}(\boldsymbol{F})=\mathrm{J}(\boldsymbol{F})+b \mathrm{~J}(\boldsymbol{F})$ are given, where $\mathrm{J}(\boldsymbol{F})$ denotes the subgroup consisting of the elements preserving the direction of the field $\boldsymbol{F}(\mathrm{J}(\boldsymbol{F}): \boldsymbol{F} \rightarrow \boldsymbol{F})$, while $b \mathrm{~J}(\boldsymbol{F})$ is a set of elements of the group $\mathrm{K}$ which reverse the direction of the field $\boldsymbol{F}(b \mathrm{~J}(\boldsymbol{F}): \boldsymbol{F} \rightarrow-\boldsymbol{F})$. For each group $\mathrm{K}(\boldsymbol{F})$ - the number is given, which shows where, in Table III one can read off the explicit form of the real part of tensor $\varepsilon_{i j}$, or, more precisely, its even part $\varepsilon_{i j}^{\mathrm{e}}\left(\varepsilon_{i j}^{\mathrm{e}}(\omega, \boldsymbol{F})=\varepsilon_{i j}^{\mathrm{e}}(\omega,-\boldsymbol{F})\right)$ and odd part $\varepsilon_{i j}^{\circ}\left(\varepsilon_{i j}^{o}(\omega, F)=-\varepsilon_{i j}^{o}(\omega,-F)\right)$. The imaginary parts $\varepsilon_{i j}^{\prime \mathrm{e}}$ and $\varepsilon_{i j}^{\prime o}$ have exactly the same forms as $\varepsilon_{i j}^{\mathrm{e}}$ and $\varepsilon_{i j}^{\circ}$, respectively; the Kramers-Kronig relations 
say that if e.g. $\varepsilon_{12}^{\mathrm{e}} \neq 0$ then also $\varepsilon_{12}^{\prime \mathrm{e}} \neq 0$ but it does not imply that $\varepsilon_{12}^{\mathrm{e}}=\varepsilon_{12}^{\prime \mathrm{e}}$. In Table III we give also the form of the real part of the tensor $\tilde{\varepsilon}_{i j}$ for all 122 point groups (cp. Table I). They are written out in the crystalographic frame of axes. If we need to determine the difference $\tilde{\varepsilon}_{i j}(\omega, F)-\tilde{\varepsilon}_{i j}$, we must transform the latter tensor to the local frame of axes in which the tensor $\tilde{\varepsilon}_{i j}(\omega, F)$ is written out.

The susceptibility tensor $\tilde{\chi}_{i j}$ satisfies exactly the same Onsager relation [4]:

$$
\tilde{\chi}_{i j}(\omega, \boldsymbol{E}, \boldsymbol{H}, \boldsymbol{k})=\tilde{\chi}_{j i}(\omega, \boldsymbol{E},-\boldsymbol{H},-\boldsymbol{k})
$$

as the dielectric parmeability tensor:

$$
\tilde{\varepsilon}_{i j}(\omega, \boldsymbol{E}, \boldsymbol{H}, \boldsymbol{k})=\tilde{\varepsilon}_{j i}(\omega, \boldsymbol{E},-\boldsymbol{H},-k) .
$$

Therefore, the tensor $\tilde{\chi}_{i j}$ has exactly the same form as $\tilde{\varepsilon}_{i j}$. The identity of the forms of tensors $\tilde{\chi}_{i j}$ and $\tilde{\varepsilon}_{i j}$ does not mean their equivalence which would imply that for each two arbitrarily chosen components the conditions $\varepsilon_{i j} / \varepsilon_{k l}=\chi_{i j} / \chi_{k l}$ had to be fulfilled. So, as far as the forms are concerned, the Tables I-III can be also referred to the $\tilde{\chi}_{i j}(\omega, \boldsymbol{E}, \boldsymbol{H}, \boldsymbol{k})$ tensors. The analogies in the behaviour of the tensors $\varepsilon_{i j}$ and $\chi_{i j}$ in the most essential aspects of our theory (i.e. in the vicinity of the Néel, Curie temperatures and the point where the group $\mathrm{K}$ is broken to $K(F)$ group) are depicted in elegant form on Figs. 2 and 3 of the paper [5]. On the figures the angle of rotation of polarization plane of the light and the magnetic susceptibility are plotted as functions of the magnetic field.

We can obtain the information about the transport properties of the crystal also by measuring the $\chi_{i j}$ tensor [6]. In the paper [6] the tensors $\chi_{i j}$ are measured on the left- and right-hand side of Néel points. A different behaviour has been obtained. It is obvious that for two different point groups $\mathrm{K}$ we obtain the different $\mathrm{K}(\boldsymbol{H})$ groups (see: Table I and Table II) and consequently the different tensors $\tilde{\chi}_{i j}$ (and similar $\tilde{\varepsilon_{i j}}$ ) are measured.

The investigations of the phase transitions by parallel optical and magnetical methods have been announced for some time $[7,8]$.There is a great deal of hope that the Tables I-III will appear useful in such experiments.

Each medium is less or more dispersive. Therefore, while discussing for example the electrooptical effects it won't do any harm to treat the gyration tensor, $\tilde{\varepsilon}_{i j}(\omega, k)$, as the correction to the $\tilde{\varepsilon}_{i j}(\omega, E)$ tensor [9]. Table II allows to discuss these and similar effects as magnetoelectric optical effects $[5,7,8,10]$, the magnetooptical effects in dispersive medium, and so on. The theoretical analyses [9, $11]$ and the interpretations of experimental data given by experimenters $[5,12,13]$ have been based up to the present on the tables given in the monographs [14] (see: Refs. [9, 11-13]) and [15] (see: Ref. [5]).

In this paper we will discuss only the electrooptical effects. The discussion will be short as we have some analogies with previously discussed magnetooptical effects [1] and the effects following from the dispersion of wave vector $k$ in medium $[2,3]$. Since the time the papers [1,2] have appeared, there have been no new theoretical and experimental ideas, so there is no need to supplement the previous discussions $[1,2]$. 


\section{The electrooptical effects}

The tensors $\tilde{\varepsilon}_{i j}(\omega, E)$ (i.e. $\tilde{\varepsilon}_{i j}^{e}(\omega, E)$ and $\tilde{\varepsilon}_{i j}^{\circ}(\omega, E)$ - the particular cases of the tensor (2) - are obtained in exactly the same way as the tensor $\tilde{\varepsilon}_{i j}(\omega, H)[1]$ and $\tilde{\varepsilon}_{i j}(\omega, k)$ [2]. When adopting the previously described group-theoretical procedure we must remember that the field $\boldsymbol{E}$ is invariant under the action of the following groups $\mathrm{J}(E)=1,2, m, m m 2,3,3 m, 4,4 m m, 6,6 m m, 11^{\prime}, 21^{\prime}, m 1^{\prime}, m m 21^{\prime}, 31^{\prime}, 3 m 1^{\prime}$, $41^{\prime}, 4 m m 1^{\prime}, 61^{\prime}, 6 m m 1^{\prime}, 2^{\prime}, m^{\prime}, m^{\prime} m 2^{\prime}, m^{\prime} m^{\prime} 2,3 m^{\prime}, 4^{\prime}, 4^{\prime} m m^{\prime}, 4 m^{\prime} m^{\prime}, 6^{\prime}, 6^{\prime} m m^{\prime}$, $6 m^{\prime} m^{\prime}$. The results obtained in this way are not in contradiction to experimental data $[5-8,10,12,13,16,17]$.

The nonreciprocal rotation of the plane of light polarization discussed in the papers $[5,7,8,10]$, which is induced by electric field in $\mathrm{Cr}_{2} \mathrm{O}_{3}$ crystal (the symmetry group $\mathrm{K}=\overline{3}^{\prime} m^{\prime}$ ) for geometry $k\|E\| z$ (then $\mathrm{K}(\boldsymbol{E})=\overline{\mathbf{3}}^{\prime} m^{\prime}$ ) can be observed not only for $\mathrm{K}(\boldsymbol{E})=\overline{3}^{\prime} m^{\prime}$ but also when $\mathrm{K}(\boldsymbol{E})=\overline{3}^{\prime} 32, \overline{3}^{\prime} m^{\prime}, 4 / \mathrm{m}^{\prime}, 422$, $4 / \mathrm{m}^{\prime} \mathrm{m}^{\prime} \mathrm{m}^{\prime}, \overline{6}^{\prime}, 6 / \mathrm{m}$ ', $622, \overline{6}^{\prime} \mathrm{m}^{\prime} 2,6 / \mathrm{m}$ 'm'm'; the reciprocal rotation can be observed for $\mathrm{K}(\boldsymbol{E})=\overline{3}, 32^{\prime}, \overline{3} m^{\prime}, 4 / m, 42^{\prime} 2^{\prime}, 4 / m m^{\prime} m^{\prime}, \overline{6}, 6 / m, 62^{\prime} 2^{\prime}, \overline{6} 2^{\prime} m^{\prime}, 6 / m^{\prime} m^{\prime}$. In the former case the angle of rotation of polarization plane satysfies the condition:

$$
\varphi(\boldsymbol{E})=-\varphi(-\boldsymbol{E}), \quad \text { i.e. } \varphi(\boldsymbol{E}) \sim|\boldsymbol{E}| \operatorname{sign}(\boldsymbol{E}),
$$

while in the latter case - the condition

$$
\varphi(E)=\varphi(-E), \quad \text { i.e. } \varphi(\boldsymbol{E}) \sim \boldsymbol{E}^{2} .
$$

The remark: for nonreciprocal rotation the relation (4) but not (3) should be valid, while for reciprocal one - vice versa, i.e. the relation (3) but not (4) is valid; note that if the left-hand side of the relations (3) and (4) corresponds to the geometry $\pm \boldsymbol{k}\|\boldsymbol{E}\| z$ then the right-hand side of this relations corresponds to the geometry $\pm k\|-E\| z$. In turn, the experimental definitions are based on the geometries $\pm k\|\boldsymbol{E}\| z$ and $\mp k\|\boldsymbol{E}\| z$, respectively. We see that the common elements of theoretical sugestion and experimental definitions are the mutual relation between the vector $\boldsymbol{k}$ and $\boldsymbol{E}$.

It follows from our results that the nonreciprocal rotation in $\mathrm{Y}_{3} \mathrm{Fe}_{5} \mathrm{O}_{12}$ for the geometry $\boldsymbol{k}\|\boldsymbol{E}\| \boldsymbol{H} \| \boldsymbol{z}$, observed in experiments $[5,7,8]$, is generated by the magnetic field $\boldsymbol{H}$ and not by the electric one $\boldsymbol{E}$. Remark: it follows from theoretical definition of nonreciprocal and reciprocal rotations of the plane of light polarization that the external magnetic field $\boldsymbol{H}$ can only generate the reciprocal rotation, while the electric field $\boldsymbol{E}$ and the spatial dispersion of wave vector $k$ can generate both rotations, i.e. the nonreciprocal and reciprocal ones (cp. Table II and III).

For groups $\mathrm{K}(\boldsymbol{E})$ different from the above mentioned ones (e.g. $\mathrm{K}(\boldsymbol{E})=$ $m^{\prime} m^{\prime} m, m^{\prime} m m^{\prime}, m m^{\prime} m^{\prime}, m^{\prime} m^{\prime} m^{\prime}, m m^{\prime} m, m^{\prime} m m$, and so on) or for $k \not h E$ we are dealing with more complicated functions for $\varphi(\boldsymbol{E})$ than those given by formulae (3) and (4); however, also for them the first term of power series can sometimes take the form (3) or (4). The form of the above function depends not only on the symmetry but also on the relations between the components of the tensor 
$\tilde{\varepsilon}_{i j}(\omega, E)$. For the same group $\mathrm{K}(\boldsymbol{E})$ and two media we can obtain two different functions $\varphi(E)$. It is not useful to discuss the all possible relations between the elements of $\tilde{\varepsilon}_{i j}(\omega, E)$. It is more easy to interpretate the experimental data than to predict them. The group-theoretical method does not allow us to estimate which of the two functions allowed by the symmetry is more likely to be a proper one.

If $\mathrm{J}(E)=3 m, 4 m m, 6 m m, 31^{\prime}, 3 m 1^{\prime}, 41^{\prime}, 4 m m 1^{\prime}, 61^{\prime}, 6 m m 1^{\prime}, 4^{\prime}, 6^{\prime}$, $4^{\prime} \mathrm{mm}^{\prime}, 6^{\prime} \mathrm{mm}^{\prime}$ or $\mathrm{K}(\boldsymbol{E})=\overline{3} m, 4 / \mathrm{mmm}, 6 / \mathrm{mmm}, \overline{6} 2 m, \overline{3} 1^{\prime}, 321^{\prime}, \overline{3} m 1^{\prime}$, $4 / m 1^{\prime}, 4221^{\prime}, 4 / m m m 1^{\prime}, \overline{6} 1^{\prime}, 6 / m 1^{\prime}, 6221^{\prime}, \overline{6} 2 m 1^{\prime}, 6 / m m m 1^{\prime}, \overline{3}^{\prime} m, 4^{\prime} / m, 4^{\prime} / m^{\prime}$, $4^{\prime} 22^{\prime}, 4 / m^{\prime} m m, 4^{\prime} / m m m^{\prime}, 4^{\prime} / m^{\prime} m^{\prime} m, 6^{\prime} / m^{\prime}, 6^{\prime} / m, 6^{\prime} 22^{\prime}, \overline{6}^{\prime} m 2^{\prime}, 6 / m^{\prime} m m$, $6^{\prime} / \mathrm{mmm}^{\prime}, 6^{\prime} / \mathrm{m}^{\prime} m^{\prime} m$ then the $z$-axis is, similarly as in the case $\boldsymbol{E}=0$, the optical axis; it means that birefringence $\Delta n=n_{1}-n_{2}$ equals zero:

$$
\Delta n=0 \text {. }
$$

If $\boldsymbol{k} \backslash \boldsymbol{E}$ then we have

$$
\Delta n=f\left(E^{2}\right)
$$

and the first term of power series can take the form

$$
\Delta n \sim|E|
$$

or

$$
\Delta n \sim E^{2} .
$$

For the groups $\mathrm{K}(E)=2_{x} / m_{x}, 2_{x}^{\prime} / m_{x}^{\prime}, 2_{x} / m_{x} 1^{\prime}, 2_{y} / m_{y}, 2_{y}^{\prime} / m_{y}^{\prime}, 2_{y} / m_{y} 1^{\prime}, 2_{z} / m_{z}$, $2_{z}^{\prime} / m_{z}^{\prime}, 2_{z} / m_{z} 1^{\prime}, m m m, m m m^{\prime}, m m m 1^{\prime}$ the function (6), and consequently (7) or (8) will also appear for any geometry of vector $k$ with respect to the field $\boldsymbol{E}$. The quadratic $[16,17]$ and linear $[12,13]$ dependences of the field $\boldsymbol{E}$ belong to the most frequently measured.

The first term of power series for $\mathrm{K}(E)=\overline{4}, 4^{\prime}, \overline{4} 1^{\prime}, \overline{4} 2 m, \overline{4} 2 m 1^{\prime}, \overline{4} 2^{\prime} m^{\prime}, \overline{4}^{\prime} 2 m^{\prime}$, $\overline{4}^{\prime} m 2^{\prime}$ takes the form

$$
\Delta n \sim|\boldsymbol{E}| \operatorname{sign}(\boldsymbol{E})
$$

for $k \perp E \| z$, but in the case $K(E)=\overline{4}^{\prime} m 2^{\prime}-$ also for $k\|\boldsymbol{E}\| z$.

In the case of other groups $K(\boldsymbol{E})$ or for arbitrary geometry of the vector $k$ with respect to the field $E$ the form of the function $\Delta n(E)$ is determined not only by symmetry but also by the material constants. These functions are more complicated than (7), (8) or (9). For such a case it is more easy to consider the concrete experimental data than to discuss it in general form.

\section{Acknowledgments}

I wish to thank Drs P. Kosiński and M. Majewski for usueful discussions and reading the manuscript. 
TABLE I

The symmetry group $\mathrm{K}(\boldsymbol{F})$ broken by a field $\boldsymbol{F}$, where $\boldsymbol{F}$ stands for electric field $(E)$, magnetic field $(\boldsymbol{H})$ or spatial dispersion of the wave vector $(k)$ for all directions: parailel (II), perpendicular $(\perp)$ and arbitrarily oriented (a.o.) relative to the axes and planes of the K-symmetric crystal. For these $\mathrm{K}$ groups the explicit expressions of dielectric permittivity tensors $\varepsilon_{i j}$ are also given (details in Table III).

The indices $m=x, y, z ; r=x, y ; s=a, b ; i=1,2,3 ; j=1,2,3,4$ and $\mathrm{p}=\mathrm{a}$, $b, c, d, e, f$. The $\Theta$ is the element if the time inversion. The symbol $\| C_{2 m}$ is equivalent to: $\boldsymbol{F} \| \mathrm{C}_{2 x}$ or $\boldsymbol{F} \| \mathrm{C}_{2 y}$ or $\boldsymbol{F} \| \mathrm{C}_{2 z}$. The similar abbreviations are used in other cases. The number put in the column (3) enables us to reconstruct the explicit form of $\varepsilon_{i j}$ (via Table III). The groups $\mathrm{K}(\boldsymbol{F})$ are always written in the local frame of axes such that $z \| F$.

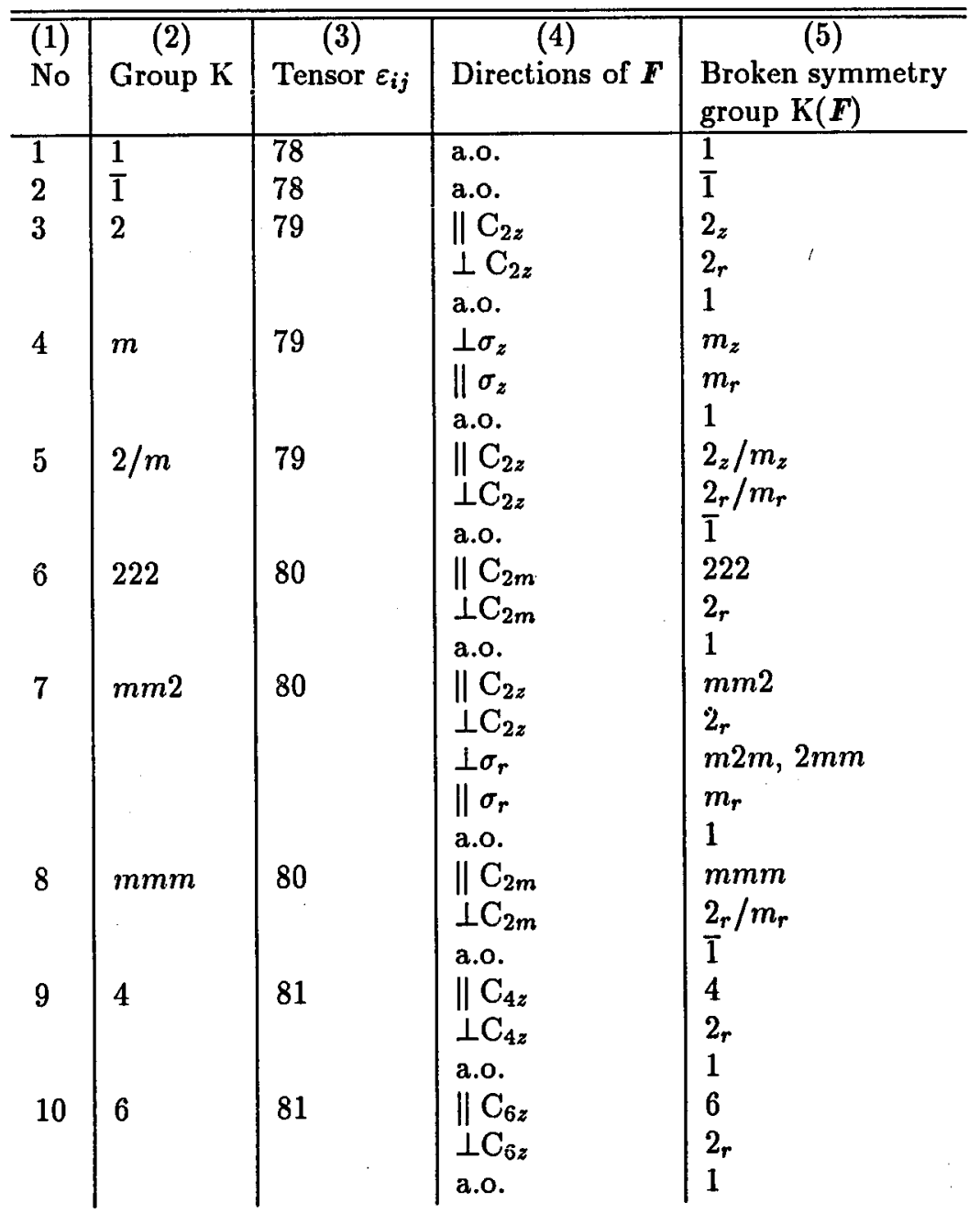


TABLE I cont.

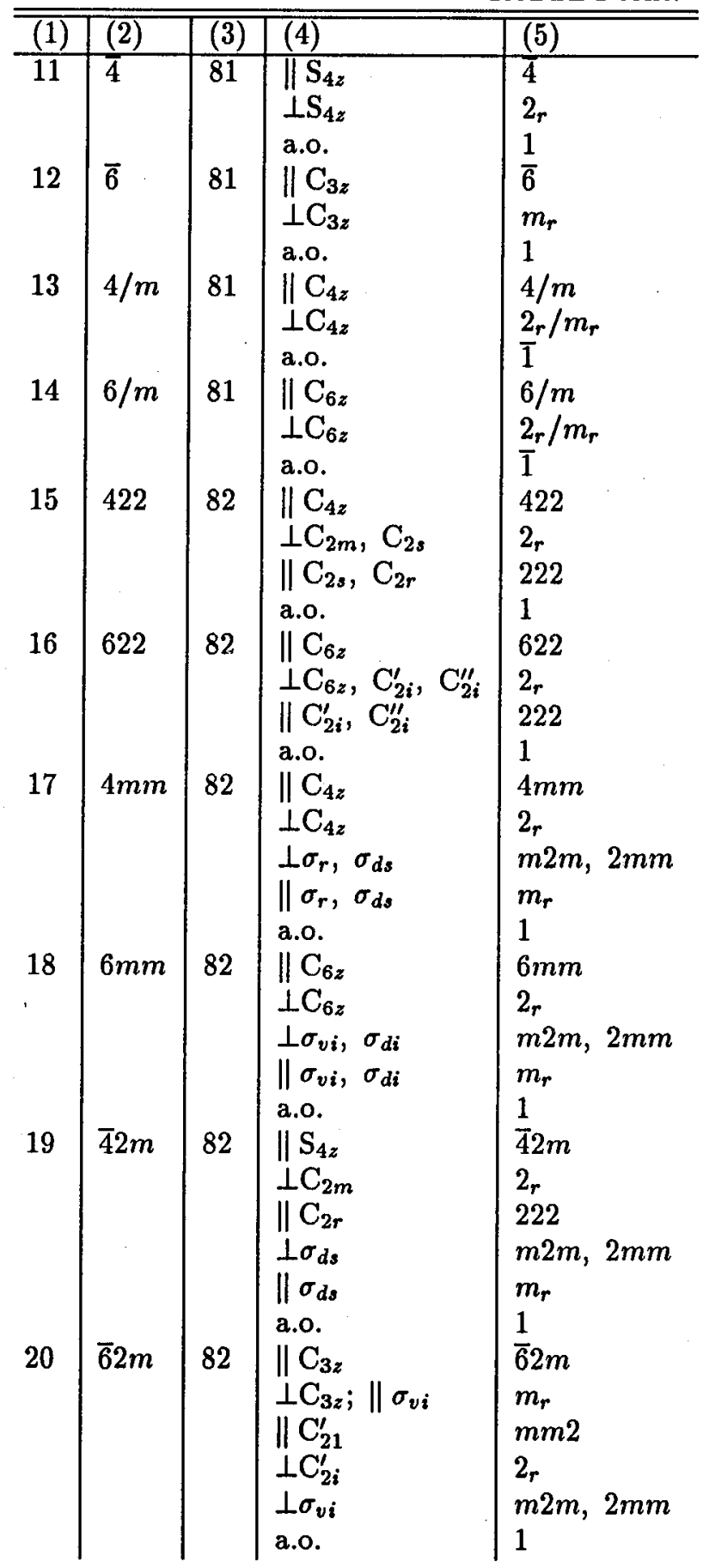


TABLE I cont.

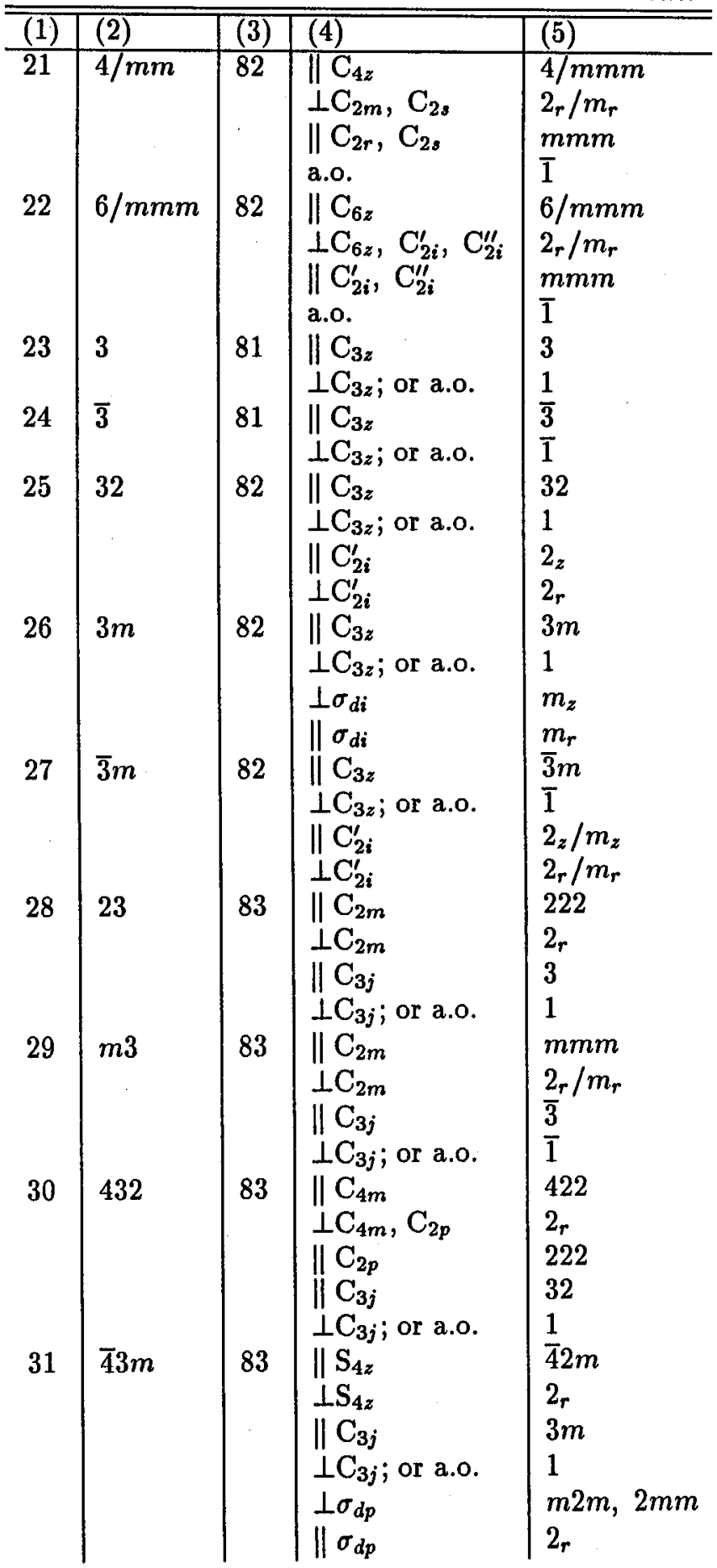


TABLE I cont.

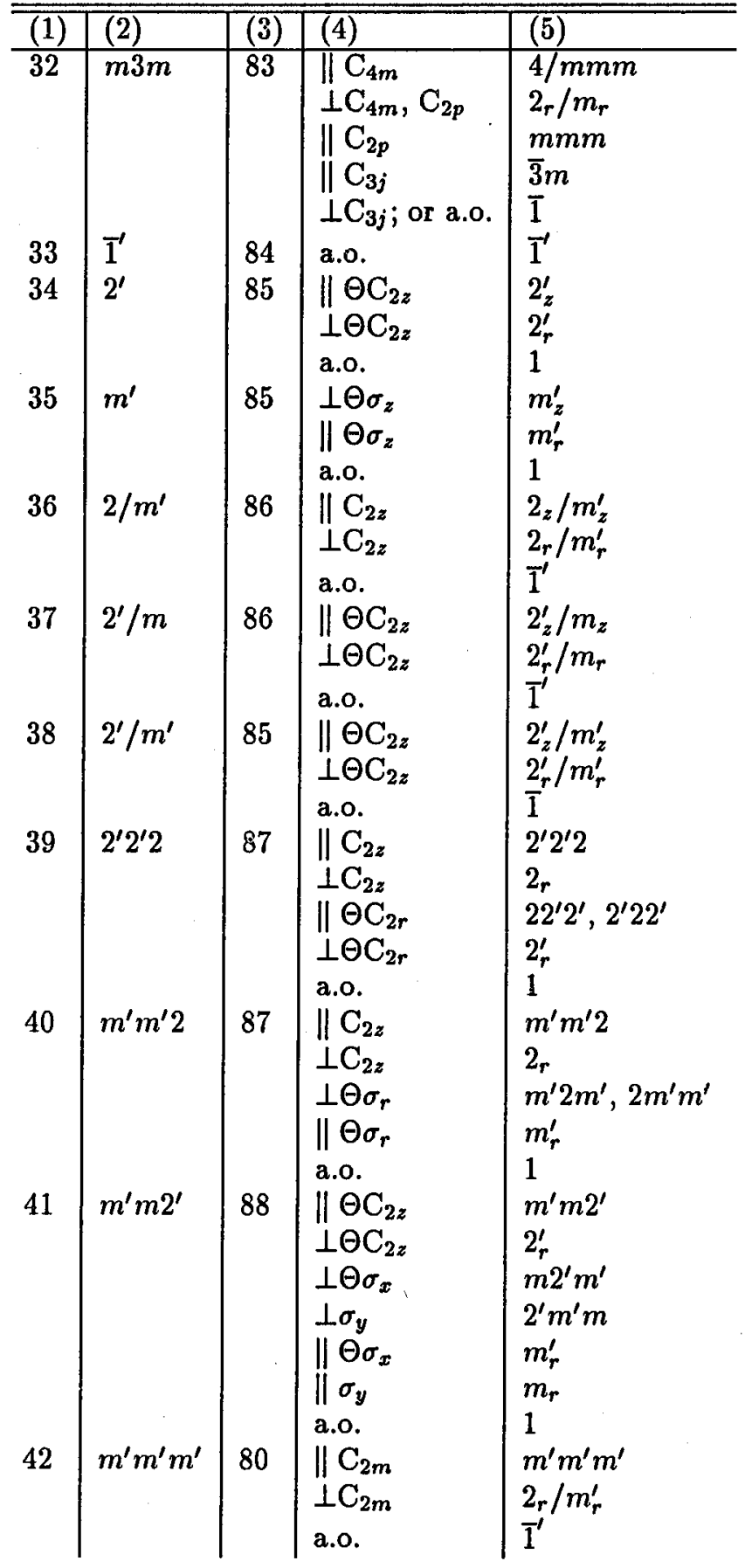


TABLE I cont.

\begin{tabular}{|c|c|c|c|c|}
\hline$\overline{\overline{(1)}}$ & $\overline{(2)}$ & (3) & (4) & $\overline{(5)}$ \\
\hline 43 & $m m m^{\prime}$ & 80 & $\begin{array}{l}\| C_{2 z} \\
\perp C_{2 z} \\
\perp \Theta C_{2 r} \\
\| \Theta C_{2 r} \\
\text { a.o. }\end{array}$ & $\begin{array}{l}m m m^{\prime} \\
2_{r} / m^{\prime} \\
2_{r}^{\prime} / m_{r} \\
m^{\prime} m m, m m^{\prime} m \\
\overline{1}^{\prime}\end{array}$ \\
\hline 44 & $m^{\prime} m^{\prime} m$ & 87 & $\begin{array}{l}\| C_{2 z} \\
\perp C_{2 z} \\
\| \Theta C_{2 r} \\
\perp \Theta C_{2 r} \\
\text { a.o. }\end{array}$ & $\begin{array}{l}m^{\prime} m^{\prime} m \\
2_{r} / m_{r} \\
m^{\prime} m m^{\prime}, m m^{\prime} m^{\prime} \\
2_{r}^{\prime} / m_{r}^{\prime}\end{array}$ \\
\hline 45 & $4^{\prime}$ & 82 & $\begin{array}{l}\| \Theta \mathrm{C}_{4 z} \\
\perp \Theta \mathrm{C}_{4 z} \\
\text { a.o. }\end{array}$ & $\begin{array}{l}4^{\prime} \\
2_{r} \\
1\end{array}$ \\
\hline 46 & $6^{\prime}$ & 82 & $\begin{array}{l}\| \Theta \mathrm{C}_{6 z} \\
\perp \Theta \mathrm{C}_{6 z} \\
\text { a.o. }\end{array}$ & $\begin{array}{l}6^{\prime} \\
2_{r}^{\prime} \\
1\end{array}$ \\
\hline 47 & $\overline{4}^{\prime}$ & 82 & $\begin{array}{l}\| \Theta \mathrm{S}_{4 z} \\
\perp \Theta \mathrm{S}_{4 z} \\
\text { a.o. }\end{array}$ & $\begin{array}{l}\overline{4}^{\prime} \\
2_{r} \\
1\end{array}$ \\
\hline 48 & $\overline{6}^{\prime}$ & 82 & $\begin{array}{l}\| \Theta S_{3 z} \\
\perp \Theta S_{3 z} \\
\text { a.o. }\end{array}$ & $\begin{array}{l}\overline{6}^{\prime} \\
m_{r}^{\prime} \\
1\end{array}$ \\
\hline 49 & $42^{\prime} 2^{\prime}$ & 81 & $\begin{array}{l}\| \mathrm{C}_{4 z} \\
\perp \mathrm{C}_{4 z} \\
\| \Theta \mathrm{C}_{2 r}, \Theta \mathrm{C}_{2 s} \\
\perp \Theta \mathrm{C}_{2 r}, \Theta \mathrm{C}_{2 s} \\
\text { a.o. }\end{array}$ & $\begin{array}{l}42^{\prime} 2^{\prime} \\
2_{r} \\
2^{\prime} 22^{\prime}, 22^{\prime} 2^{\prime} \\
2_{r}^{\prime} \\
1\end{array}$ \\
\hline 50 & $62^{\prime} 2^{\prime}$ & 81 & $\begin{array}{l}\| C_{6 z} \\
\perp C_{6 z} \\
\| \Theta C_{2 i}^{\prime}, \Theta C_{2 i}^{\prime} \\
\perp \Theta C_{2 i}^{\prime}, \Theta C_{2 i}^{\prime \prime} \\
\text { a.o. }\end{array}$ & $\begin{array}{l}62^{\prime} 2^{\prime} \\
2_{r} \\
2^{\prime} 22^{\prime}, 22^{\prime} 2^{\prime} \\
2_{r}^{\prime} \\
1\end{array}$ \\
\hline 51 & $4^{\prime} 22^{\prime}$ & 82 & $\begin{array}{l}\| \Theta \mathrm{C}_{4 z} \\
\perp \mathrm{C}_{2 m} \\
\| \mathrm{C}_{2 r} \\
\| \Theta \mathrm{C}_{2 s} \\
\perp \Theta \mathrm{C}_{2 s} \\
\text { a.o. }\end{array}$ & $\begin{array}{l}4^{\prime} 22^{\prime} \\
2_{r} \\
222 \\
2^{\prime} 22^{\prime}, 22^{\prime} 2^{\prime} \\
2_{r}^{\prime} \\
1\end{array}$ \\
\hline 52 & $6^{\prime} 22^{\prime}$ & 82 & $\begin{array}{l}\| \Theta \mathrm{C}_{6 z} \\
\perp \Theta \mathrm{C}_{2 z}, \Theta \mathrm{C}_{2 i}^{\prime} \\
\| \Theta \mathrm{C}_{2 i}^{\prime} \\
\| \mathrm{C}_{2 i}^{\prime \prime} \\
\perp \mathrm{C}_{2 i}^{\prime \prime} \\
\text { a.o. }\end{array}$ & $\begin{array}{l}6^{\prime} 22^{\prime} \\
2_{r}^{\prime} \\
2^{\prime} 22^{\prime}, 22^{\prime} 2^{\prime} \\
2^{\prime} 2^{\prime} 2 \\
2_{r} \\
1\end{array}$ \\
\hline
\end{tabular}


TABLE I cont.

\begin{tabular}{|c|c|c|c|c|}
\hline$\overline{(1)}$ & $\overline{(2)}$ & $\overline{(3)}$ & $\overline{(4)}$ & $\overline{(5)}$ \\
\hline 53 & $4 / m^{\prime}$ & 82 & $\begin{array}{l}\| \mathrm{C}_{4 z} \\
\perp \mathrm{C}_{4 z} \\
\text { a.o. }\end{array}$ & $\begin{array}{l}4 / m^{\prime} \\
2 r / m_{r}^{\prime} \\
\overrightarrow{1}^{\prime}\end{array}$ \\
\hline 54 & $6 / m^{\prime}$ & 82 & $\begin{array}{l}\| \mathrm{C}_{6 z} \\
\perp \mathrm{C}_{6 z} \\
\text { a.o. }\end{array}$ & $\begin{array}{l}6 / m^{\prime} \\
2 / m_{r}^{\prime} \\
\overrightarrow{1}\end{array}$ \\
\hline 55 & $4^{\prime} / m^{\prime}$ & 82 & $\begin{array}{l}\| \Theta \mathrm{C}_{4 z} \\
\perp \Theta \mathrm{C}_{4 z} \\
\text { a.o. }\end{array}$ & $\begin{array}{l}4^{\prime} / m^{\prime} \\
2_{r} / m_{r}^{\prime} \\
\overrightarrow{1}\end{array}$ \\
\hline 56 & $6^{\prime} / m$ & 82 & $\begin{array}{l}\| \Theta_{6 z} \\
\perp \Theta C_{6 z} \\
\text { a.o. }\end{array}$ & $\begin{array}{l}6^{\prime} / m \\
2_{r}^{\prime} / m_{r} \\
\overrightarrow{1}\end{array}$ \\
\hline 57 & $4^{\prime} / m$ & 82 & $\begin{array}{l}\| \Theta \mathrm{C}_{4 z} \\
\perp \Theta \mathrm{C}_{4 z} \\
\text { a.o. }\end{array}$ & $\begin{array}{l}4^{\prime} / m \\
2_{r} / m_{r} \\
1\end{array}$ \\
\hline 58 & $6^{\prime} / m^{\prime}$ & 82 & $\begin{array}{l}\| \Theta \mathrm{C}_{6 z} \\
\perp \Theta \mathrm{C}_{6 z} \\
\text { a.o. }\end{array}$ & $\begin{array}{l}6^{\prime} / m^{\prime} \\
\frac{2}{r} / m_{r}^{\prime} \\
\frac{1}{1}\end{array}$ \\
\hline 59 & $4 m^{\prime} m^{\prime}$ & 81 & $\begin{array}{l}\| \mathrm{C}_{4 z} \\
\perp \mathrm{C}_{4 z} \\
\| \Theta \sigma_{r}, \Theta \sigma_{d s} \\
\perp \Theta \sigma_{r}, \Theta \sigma_{d s} \\
\text { a.o. }\end{array}$ & $\begin{array}{l}4 m^{\prime} m^{\prime} \\
2 r \\
m_{r}^{\prime} \\
m^{\prime} 2 m^{\prime}, 2 m^{\prime} m^{\prime} \\
1\end{array}$ \\
\hline 60 & $6 m^{\prime} m^{\prime}$ & 81 & $\begin{array}{l}\| \mathrm{C}_{6 z} \\
\perp \mathrm{C}_{6 z} \\
\| \Theta \sigma_{v i}, \Theta \sigma_{d i} \\
\perp \Theta \sigma_{v i}, \Theta \sigma_{d i} \\
\text { a.o. }\end{array}$ & $\begin{array}{l}6 m^{\prime} m^{\prime} \\
2 r \\
m_{r}^{\prime} \\
m^{\prime} 2 m^{\prime}, 2 m^{\prime} m^{\prime} \\
1\end{array}$ \\
\hline 61 & $4^{\prime} \mathrm{mm}^{\prime}$ & 82 & $\begin{array}{l}\| \Theta \mathrm{C}_{4 z} \\
\perp \Theta \mathrm{C}_{4 z} \\
\| \sigma_{r} \\
\perp \sigma_{r} \\
\| \Theta \sigma_{d s} \\
\perp \Theta \sigma_{d s} \\
\text { a.o. }\end{array}$ & $\begin{array}{l}4^{\prime} m m^{\prime} \\
2 r \\
m_{r} \\
2 m m, \quad m 2 m \\
m_{r}^{\prime} \\
m^{\prime} 2 m^{\prime}, 2 m^{\prime} m^{\prime} \\
1\end{array}$ \\
\hline 62 & $6^{\prime} m^{\prime} m$ & 82 & $\begin{array}{l}\| \Theta C_{6 z} \\
\perp \Theta C_{6 z} \\
\| \sigma_{d i} \\
\perp \sigma_{d i} \\
\| \Theta \sigma_{v i} \\
\perp \Theta \sigma_{v i} \\
\text { a.o. }\end{array}$ & $\begin{array}{l}6^{\prime} m^{\prime} m \\
2_{r}^{\prime} \\
m_{r} \\
2^{\prime} m^{\prime} m, m^{\prime} 2^{\prime} m \\
m_{r}^{\prime} \\
2^{\prime} m m^{\prime}, m 2^{\prime} m^{\prime} \\
1\end{array}$ \\
\hline
\end{tabular}


TABLE I cont.

\begin{tabular}{|c|c|c|c|c|}
\hline (1) & (2) & $(3)$ & $(4)$ & (5) \\
\hline 63 & $\overline{42^{\prime} m^{\prime}}$ & 81 & $\begin{array}{l}\| S_{4 z} \\
\perp S_{4 z} \\
\| \Theta C_{2 r} \\
\perp \Theta C_{2 r} \\
\| \Theta \sigma_{d s} \\
\perp \Theta \sigma_{d s} \\
\text { a.o. }\end{array}$ & $\begin{array}{l}42^{\prime} m^{\prime} \\
2 \\
2_{r}^{\prime} 22^{\prime}, 22^{\prime} 2^{\prime} \\
2_{r}^{\prime} \\
m_{r}^{\prime} \\
2 m^{\prime} m^{\prime}, m^{\prime} 2 m^{\prime} \\
1\end{array}$ \\
\hline 64 & $\overline{6} m^{\prime} 2^{\prime}$ & 81 & $\begin{array}{l}\| S_{3 z} \\
\perp S_{3 z} \\
\| \Theta C_{2 i}^{\prime} \\
\perp \Theta C_{2 i}^{\prime} \\
\| \Theta \sigma_{v i} \\
\perp \Theta \sigma_{v i} \\
\text { a.o. }\end{array}$ & $\begin{array}{l}\overline{6} m^{\prime} 2^{\prime} \\
m_{r} \\
m m^{\prime} 2^{\prime}, m^{\prime} m 2^{\prime} \\
2_{r}^{\prime} \\
m_{r}^{\prime} \\
2^{\prime} m m^{\prime}, m 2^{\prime} m^{\prime} \\
1\end{array}$ \\
\hline 65 & $\overline{4}^{\prime} 2 m^{\prime}$ & 82 & $\begin{array}{l}\| \Theta S_{4 z} \\
\perp C_{2 m} \\
\| \mathrm{C}_{2 r} \\
\| \Theta \sigma_{d s} \\
\perp \Theta \sigma_{d s} \\
\text { a.o. }\end{array}$ & $\begin{array}{l}\overline{4}^{\prime} 2 m^{\prime} \\
2 r \\
222 \\
m_{r}^{\prime} \\
m^{\prime} 2 m^{\prime}, 2 m^{\prime} m^{\prime} \\
1\end{array}$ \\
\hline 66 & $\overline{6}^{\prime} m^{\prime} 2$ & 82 & $\begin{array}{l}\| \Theta S_{3 z} \\
\perp \Theta S_{3 z} ; \| \Theta \sigma_{v i} \\
\| C_{2 i}^{\prime} \\
\perp \Theta \sigma_{v i} \\
\perp C_{2 i}^{\prime} \\
\text { a.o. }\end{array}$ & $\begin{array}{l}\overline{6}^{\prime} m^{\prime} 2 \\
m_{r}^{\prime} \\
m^{\prime} m^{\prime} 2 \\
2 m^{\prime} m^{\prime}, m^{\prime} 2 m^{\prime} \\
2 r \\
1\end{array}$ \\
\hline 67 & $\overline{4}^{\prime} m 2^{\prime}$ & 82 & $\begin{array}{l}\| \Theta S_{4 z} \\
\perp \Theta S_{4 z} \\
\| \Theta C_{2 r} \\
\perp \Theta C_{2 r} \\
\| \sigma_{d s} \\
\perp \sigma_{d s} \\
\text { a.o. }\end{array}$ & $\begin{array}{l}\overline{4}^{\prime} m 2^{\prime} \\
2_{r} \\
2^{\prime} 22^{\prime}, 22^{\prime} 2^{\prime} \\
2_{r}^{\prime} \\
m_{r} \\
2 m m, m 2 m \\
1\end{array}$ \\
\hline 68 & $\overline{6}^{\prime} m 2^{\prime}$ & 82 & $\begin{array}{l}\| \Theta S_{3 z} \\
\perp \Theta S_{3 z} \\
\| \Theta C_{2 i}^{\prime} \\
\perp \Theta C_{2 i}^{\prime} \\
\| \sigma_{v i} \\
\perp \sigma_{v i} \\
\text { a.o. }\end{array}$ & $\begin{array}{l}\overline{6}^{\prime} m 2^{\prime} \\
m_{r}^{\prime} \\
m m^{\prime} 2^{\prime}, m^{\prime} m 2^{\prime} \\
2_{r}^{\prime} \\
m_{r} \\
m^{\prime} 2^{\prime} m, 2^{\prime} m^{\prime} m \\
1\end{array}$ \\
\hline
\end{tabular}


TABLE I cont.

\begin{tabular}{|c|c|c|c|c|}
\hline$\overline{(1)}$ & 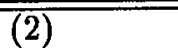 & $\overline{(3)}$ & $(4)$ & $(5)$ \\
\hline 69 & $4 / m^{\prime} m^{\prime} m^{\prime}$ & 82 & $\begin{array}{l}\| \mathrm{C}_{4 z} \\
\perp \mathrm{C}_{2 m}, \mathrm{C}_{2 s} \\
\| \mathrm{C}_{2 r}, \mathrm{C}_{2 s} \\
\text { a.o. }\end{array}$ & $\begin{array}{l}4 / m^{\prime} m^{\prime} m^{\prime} \\
2_{r} / m_{r}^{\prime} \\
m^{\prime} m^{\prime} m^{\prime} \\
\overrightarrow{1}^{\prime}\end{array}$ \\
\hline 70 & $6 / \boldsymbol{m}^{\prime} \boldsymbol{m}^{\prime} \boldsymbol{m}^{\prime}$ & 82 & $\begin{array}{l}\| \mathrm{C}_{6 z} \\
\perp \mathrm{C}_{6 z}, \mathrm{C}_{2 i}^{\prime}, \mathrm{C}_{2 i}^{\prime \prime} \\
\| \mathrm{C}_{2 i}^{\prime}, \mathrm{C}_{2 i}^{\prime \prime} \\
\text { a.o. }\end{array}$ & $\begin{array}{l}6 / m^{\prime} m^{\prime} m^{\prime} \\
2_{r} / m_{r}^{\prime} \\
m^{\prime} m^{\prime} m^{\prime} \\
\overline{1}^{\prime}\end{array}$ \\
\hline 71 & $4 / m^{\prime} m m$ & 82 & $\begin{array}{l}\| \mathrm{C}_{4 z} \\
\perp \mathrm{C}_{4 z} \\
\| \Theta \mathrm{C}_{2 r}, \Theta \mathrm{C}_{2 s} \\
\perp \Theta \mathrm{C}_{2 r}, \Theta \mathrm{C}_{2 s} \\
\text { a.o. }\end{array}$ & $\begin{array}{l}4 / m^{\prime} m m \\
2_{r} / m_{r}^{\prime} \\
m m^{\prime} m, m^{\prime} m m \\
2_{r}^{\prime} / m_{r} \\
\overline{1}^{\prime}\end{array}$ \\
\hline 72 & $6 / m^{\prime} m m$ & 82 & $\begin{array}{l}\| \mathrm{C}_{6 z} \\
\perp \mathrm{C}_{6 z} \\
\| \Theta \mathrm{C}_{2 i}^{\prime}, \Theta \mathrm{C}_{2 i}^{\prime \prime} \\
\perp \Theta \mathrm{C}_{2 i}^{\prime}, \Theta \mathrm{C}_{2 i}^{\prime \prime} \\
\text { a.o. }\end{array}$ & $\begin{array}{l}6 / m^{\prime} m m \\
2 r / m_{r}^{\prime} \\
m m^{\prime} m, m^{\prime} m m \\
2_{r}^{\prime} / m_{r} \\
\overline{1}^{\prime}\end{array}$ \\
\hline 73 & $4^{\prime} / m^{\prime} m^{\prime} m$ & 82 & $\begin{array}{l}\| \Theta \mathrm{C}_{4 z} \\
\perp \mathrm{C}_{2 m} \\
\| \mathrm{C}_{2 r} \\
\| \Theta \mathrm{C}_{2 s} \\
\perp \Theta \mathrm{C}_{2 s} \\
\text { a.o. }\end{array}$ & $\begin{array}{l}4^{\prime} / m^{\prime} m^{\prime} m \\
2_{r} / m_{r}^{\prime} \\
m^{\prime} m^{\prime} m^{\prime} \\
m m^{\prime} m, m^{\prime} m m \\
2_{r}^{\prime} / m_{r} \\
\overline{1}^{\prime}\end{array}$ \\
\hline 74 & $6^{\prime} / \mathrm{mmm}^{\prime}$ & 82 & $\begin{array}{l}\| \Theta S_{6 z} \\
\perp \Theta S_{6 z}, \Theta C_{2 i}^{\prime \prime} \\
\| \Theta C_{2 i}^{\prime \prime} \\
\| C_{2 i}^{\prime} \\
\perp C_{2 i}^{\prime} \\
\text { a.o. }\end{array}$ & $\begin{array}{l}6^{\prime} / m m m^{\prime} \\
2_{r}^{\prime} / m_{r} \\
m m^{\prime} m_{3}, m^{\prime} m m \\
m m m^{\prime} \\
2_{r} / m_{r}^{\prime} \\
\overline{1}^{\prime}\end{array}$ \\
\hline 75 & $4^{\prime} / \mathrm{mmm}^{\prime}$ & 82 & $\begin{array}{l}\| \Theta C_{4 z} \\
\perp C_{2 m} \\
\| C_{2 r} \\
\| \Theta C_{2 s} \\
\perp \Theta C_{2 s} \\
\text { a.o. }\end{array}$ & $\begin{array}{l}4^{\prime} / m m m^{\prime} \\
2_{r} / m_{r} \\
m m m \\
m^{\prime} m m^{\prime}, m m^{\prime} m^{\prime} \\
2_{r}^{\prime} / m_{r}^{\prime} \\
\frac{1}{1}\end{array}$ \\
\hline 76 & $6^{\prime} / m^{\prime} m^{\prime} m$ & 82 & $\begin{array}{l}\| \Theta \mathrm{C}_{6 z} \\
\perp \Theta \mathrm{C}_{6 z}, \Theta \mathrm{C}_{2 i}^{\prime \prime} \\
\| \Theta \mathrm{C}_{2 i}^{\prime \prime} \\
\| \mathrm{C}_{2 i}^{\prime} \\
\perp \mathrm{C}_{2 i}^{\prime} \\
\text { a.o. }\end{array}$ & $\begin{array}{l}6^{\prime} / m^{\prime} m^{\prime} m \\
2_{r}^{\prime} / m_{r}^{\prime} \\
m^{\prime} m m^{\prime}, m m^{\prime} m^{\prime} \\
m^{\prime} m^{\prime} m \\
\frac{2_{r} / m_{r}}{1}\end{array}$ \\
\hline
\end{tabular}


TABLE I cont.

\begin{tabular}{|c|c|c|c|c|}
\hline$\overline{(1)}$ & $\overline{(2)}$ & $\overline{(3)}$ & (4) & $\overline{(5)}$ \\
\hline 77 & $4 / m m^{\prime} m^{\prime}$ & 81 & $\begin{array}{l}\| \mathrm{C}_{4 z} \\
\perp \mathrm{C}_{4 z} \\
\| \Theta \mathrm{C}_{2 r}, \Theta \mathrm{C}_{2 s} \\
\perp \Theta \mathrm{C}_{2 r}, \Theta \mathrm{C}_{2 s} \\
\text { a.o. }\end{array}$ & $\begin{array}{l}4 / m m^{\prime} m^{\prime} \\
2_{r} / m_{r} \\
m^{\prime} m m^{\prime}, m m^{\prime} m^{\prime} \\
2_{r}^{\prime} / m_{r}^{\prime} \\
1\end{array}$ \\
\hline 78 & $6 / m m^{\prime} m^{\prime}$ & 81 & $\begin{array}{l}\| \mathrm{C}_{6 z} \\
\perp \mathrm{C}_{6 z} \\
\| \Theta \mathrm{C}_{2 i}^{\prime}, \Theta \mathrm{C}_{2 i}^{\prime \prime} \\
\perp \Theta \mathrm{C}_{2 i}^{\prime}, \Theta \mathrm{C}_{2 i}^{\prime \prime} \\
\text { a.o. }\end{array}$ & $\begin{array}{l}6 / m m^{\prime} m^{\prime} \\
2_{r} / m_{r} \\
m^{\prime} m m^{\prime}, m m^{\prime} m^{\prime} \\
\frac{2_{r}^{\prime} / m_{r}^{\prime}}{1}\end{array}$ \\
\hline 79 & $32^{\prime}$ & 81 & $\begin{array}{l}\| \mathrm{C}_{3 z} \\
\perp \mathrm{C}_{3 z} ; \text { or a.o. } \\
\| \Theta \mathrm{C}_{2 i}^{\prime} \\
\perp \Theta \mathrm{C}_{2 i}^{\prime}\end{array}$ & . \\
\hline 80 & $3 m^{\prime}$ & 81 & $\begin{array}{l}\| \mathrm{C}_{3 z} \\
\perp \mathrm{C}_{3 z} ; \text { or a.o. } \\
\| \Theta \sigma_{d i} \\
\perp \Theta \sigma_{d i}\end{array}$ & 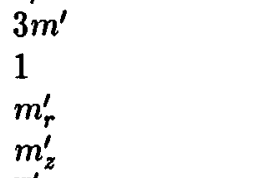 \\
\hline 81 & $\overline{3}^{\prime}$ & 82 & $\begin{array}{l}\| \Theta S_{6 z} \\
\perp \Theta S_{6 z} ; \text { or a.o. }\end{array}$ & $\begin{array}{l}\overline{3}^{\prime} \\
\overline{1}^{\prime}\end{array}$ \\
\hline 82 & $\overline{3} m^{\prime}$ & 81 & $\begin{array}{l}\| S_{6 z} \\
\perp S_{6 z} ; \text { or a.o. } \\
\| \Theta C_{2 i}^{\prime} \\
\perp \Theta C_{2 i}^{\prime}\end{array}$ & $\begin{array}{l}\overline{3} m^{\prime} \\
\overline{1} \\
2_{z}^{\prime} / m_{z}^{\prime} \\
2_{r}^{\prime} / m_{r}^{\prime}\end{array}$ \\
\hline 83 & $\overline{3}^{\prime} m$ & 82 & $\begin{array}{l}\| \Theta \mathrm{S}_{6 z} \\
\perp \Theta \mathrm{S}_{6 z} ; \text { or a.o. } \\
\| \Theta \mathrm{C}_{2 i}^{\prime} \\
\perp \Theta \mathrm{C}_{2 i}^{\prime}\end{array}$ & $\begin{array}{l}\overline{3}^{\prime} m \\
\overline{1}^{\prime} \\
2_{z}^{\prime} / m_{z} \\
2_{r}^{\prime} / m_{r}\end{array}$ \\
\hline 84 & $\overline{3}^{\prime} m^{\prime}$ & 82 & $\begin{array}{l}\| \Theta S_{6 z} \\
\perp \Theta S_{6 z} ; \text { or a.o } \\
\| C_{2 i}^{\prime} \\
\perp C_{2 i}^{\prime}\end{array}$ & $\begin{array}{l}\overline{3}^{\prime} m^{\prime} \\
\overline{1}^{\prime} \\
2_{z} / m_{z}^{\prime} \\
2_{r} / m_{r}^{\prime}\end{array}$ \\
\hline 85 & $m^{\prime} 3$ & 83 & $\begin{array}{l}\| C_{2 m} \\
\perp C_{2 m} \\
\| \Theta S_{6 j} \\
\perp \Theta S_{6 j} ; \text { or a.o. }\end{array}$ & $\begin{array}{l}m^{\prime} m^{\prime} m^{\prime} \\
2 r / m_{r}^{\prime} \\
\overline{3}^{\prime} \\
\overline{1}^{\prime}\end{array}$ \\
\hline 86 & $\overline{4}^{\prime} 3 m^{\prime}$ & 83 & $\begin{array}{l}\| \Theta S_{4 m} \\
\perp \Theta S_{4 m} \\
\| C_{3 j} \\
\perp C_{3 j} ; \text { or a.o. } \\
\| \Theta \sigma_{d p} \\
\perp \Theta \sigma_{d p}\end{array}$ & \begin{tabular}{|l}
$\overline{4}^{\prime} 2 m^{\prime}$ \\
$22_{r}$ \\
$3 m^{\prime}$ \\
1 \\
$m_{r}^{\prime}$ \\
$m^{\prime} 2 m^{\prime}, 2 m^{\prime} m^{\prime}$
\end{tabular} \\
\hline
\end{tabular}


TABLE I cont.

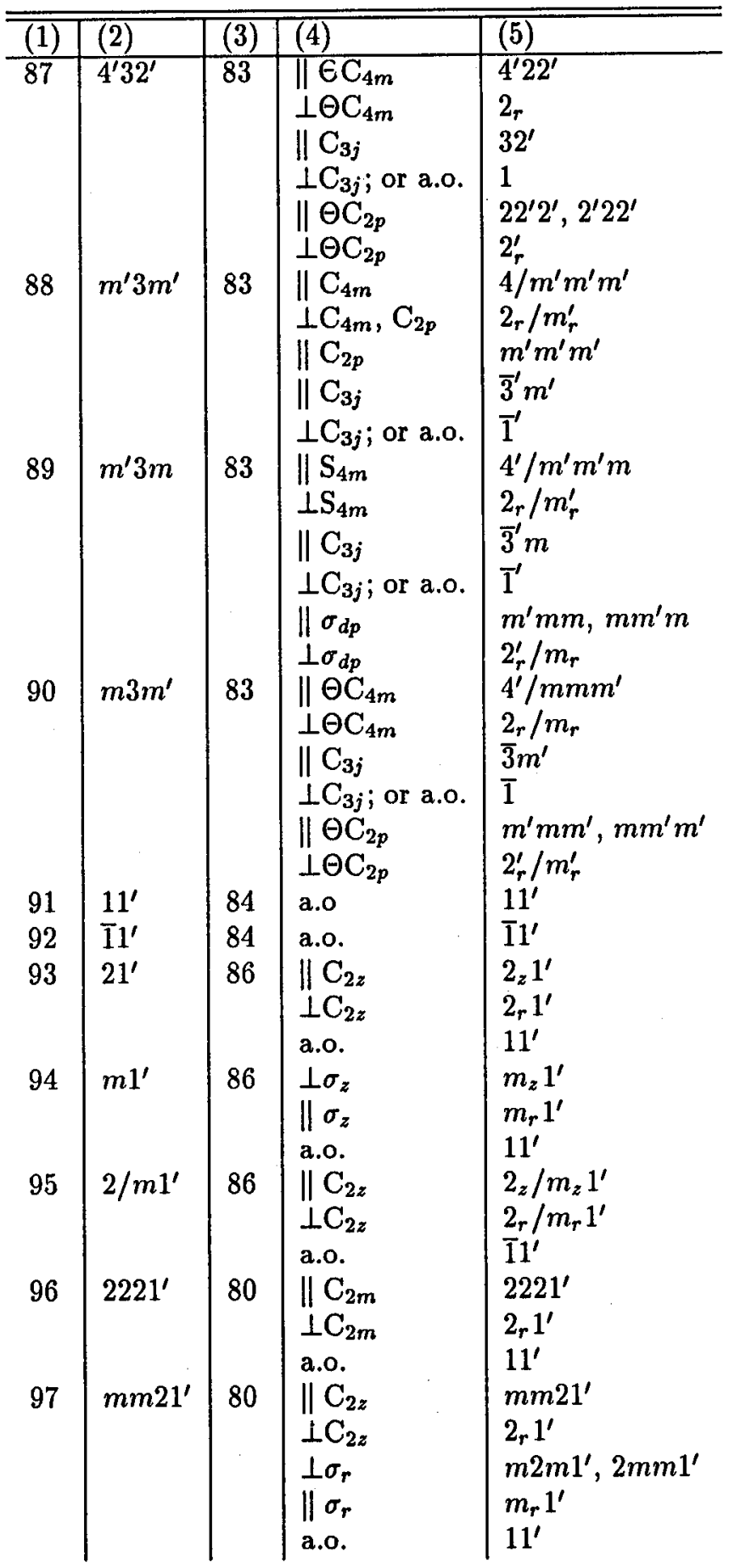


TABLE I cont.

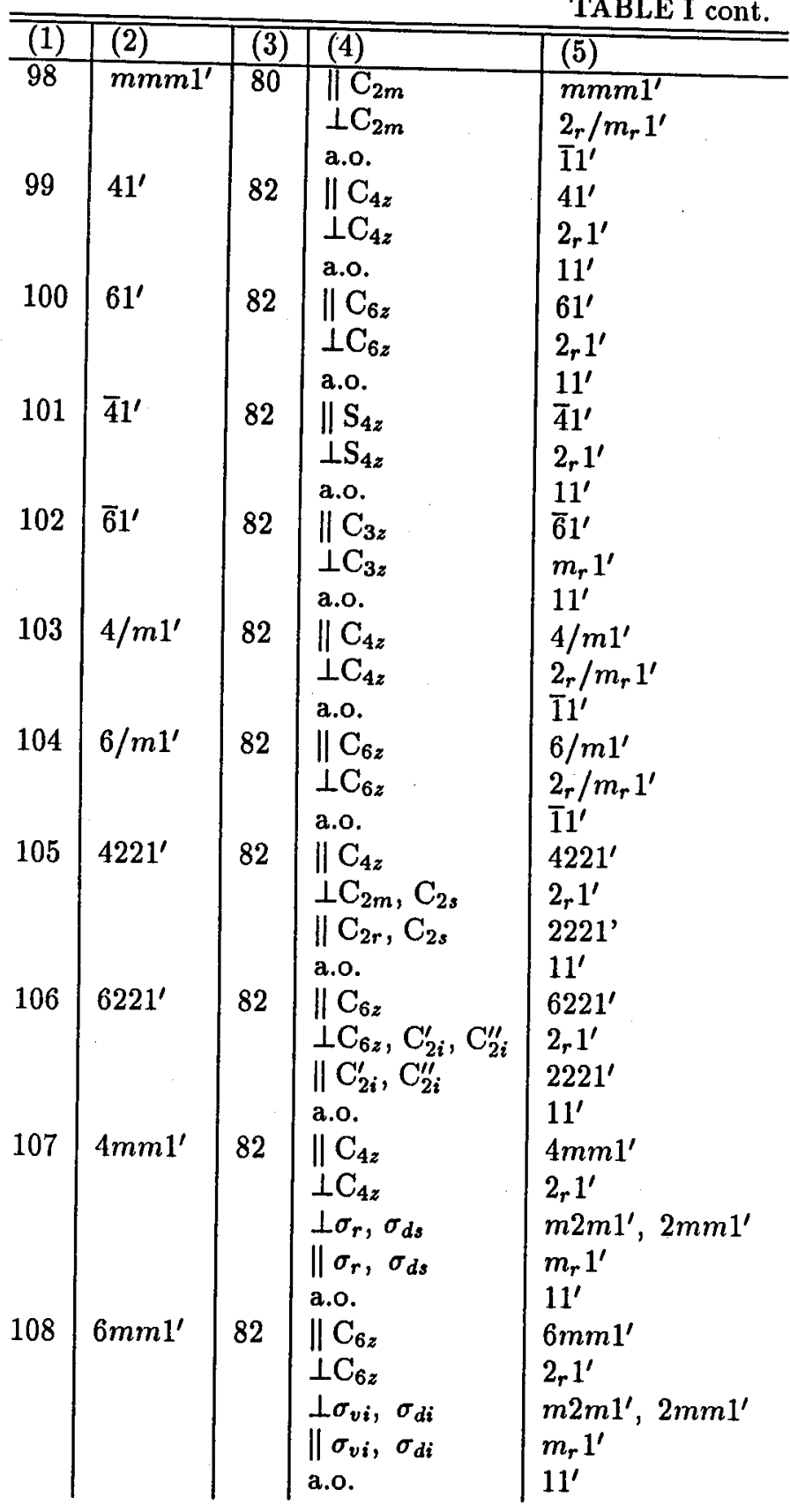


TABLE I cont.

\begin{tabular}{|c|c|c|c|c|}
\hline (1) & $\overline{(2)}$ & (3) & $\overline{(4)}$ & (5) \\
\hline \multirow[t]{4}{*}{109} & $\overline{4} 2 m 1^{\prime}$ & 82 & $\| \mathrm{S}_{4 z}$ & $42 m 1^{\prime}$ \\
\hline & & & $\begin{array}{l}\stackrel{\perp}{\perp} C_{2 m} \\
\| \sigma_{d r}\end{array}$ & $\begin{array}{l}2,1^{\prime} \\
m 2 m 1^{\prime}, 2 m m 1^{\prime}\end{array}$ \\
\hline & & & $\perp \sigma_{d r}$ & $m_{r} 1^{\prime}$ \\
\hline & & & $\| \mathrm{C}_{2 r}$ & $2221^{\prime}$ \\
\hline \multirow[t]{4}{*}{110} & $\overline{6} 2 m 1^{\prime}$ & 82 & $\begin{array}{l}\text { a.o. } \\
\| \mathrm{C}_{3 z}\end{array}$ & $\begin{array}{l}11^{\prime} \\
\overline{6} 2 m 1^{\prime}\end{array}$ \\
\hline & & & $\stackrel{\stackrel{\|}{\perp} \mathrm{C}_{3 z} ; \| \sigma_{v i}}{\| \mathrm{C}^{\prime}}$ & $\begin{array}{l}m_{\mathbf{r}} 1^{\prime} \\
m m 21^{\prime}\end{array}$ \\
\hline & & & $\perp C_{2 i}^{\prime}$ & $\begin{array}{l}m m 21 \\
2_{r} 1^{\prime}\end{array}$ \\
\hline & & & $\begin{array}{l}\perp \sigma_{v i} \\
\text { a.o. }\end{array}$ & $\begin{array}{l}m 2 m 1^{\prime}, 2 m m 1^{\prime} \\
11^{\prime}\end{array}$ \\
\hline \multirow[t]{3}{*}{111} & $4 / m m m 1^{\prime}$ & 82 & $\| \mathrm{C}_{4 z}$ & $4 / m m m 1^{\prime}$ \\
\hline & & & $\begin{array}{l}\perp \mathrm{C}_{2 m}, \mathrm{C}_{2 s} \\
\| \mathrm{C}_{2 r}, \mathrm{C}_{2 s}\end{array}$ & $\begin{array}{l}2_{r} / m_{r} 1^{\prime} \\
m m m 1^{\prime}\end{array}$ \\
\hline & $6 / m m m 1^{\prime}$ & 89 & a.o. & $\begin{array}{l}\overline{1} 1^{\prime} \\
6 / m m m 1^{\prime}\end{array}$ \\
\hline \multirow{2}{*}{112} & & 82 & $\perp \mathrm{C}_{6 z}, \mathrm{C}_{2 i}^{\prime}, \mathrm{C}_{2 i}^{\prime \prime}$ & $\begin{array}{l}2_{r} / m_{r} 1^{\prime} \\
m m m 1^{\prime}\end{array}$ \\
\hline & & & $\begin{array}{l}\| \mathrm{C}_{2 i}^{\prime}, \mathrm{C}_{2 i}^{\prime \prime} \\
\text { a.o. }\end{array}$ & $\overline{1} 1^{\prime}$ \\
\hline \multirow[t]{2}{*}{113} & $31^{\prime}$ & 82 & $\| \mathrm{C}_{3 z}$ & $31^{\prime}$ \\
\hline & & & $\perp \mathrm{C}_{3 z} ;$ or a.o. & $11^{\prime}$ \\
\hline \multirow[t]{2}{*}{114} & $\overline{3} 1^{\prime}$ & 82 & $\| \mathrm{C}_{3 z}$ & $\overline{3} 1^{\prime}$ \\
\hline & & & $\perp \mathrm{C}_{3 z} ;$ or a.o. & $11^{\prime}$ \\
\hline \multirow[t]{3}{*}{115} & $321^{\prime}$ & 82 & $\| \mathrm{C}_{3 z}$ & $321^{\prime}$ \\
\hline & & & $\frac{1 C_{3 z} ; \text { or a.o. }}{\| C^{\prime}}$ & $\begin{array}{l}11^{\prime} \\
2,1^{\prime}\end{array}$ \\
\hline & & & $\begin{array}{l}\| \mathrm{C}_{2 i}^{\prime} \\
\perp \mathrm{C}_{2 i}^{\prime}\end{array}$ & $21^{\prime}$ \\
\hline \multirow[t]{3}{*}{116} & $3 m 1^{\prime}$ & 82 & $\| \mathrm{C}_{3 z}$ & $3 m 1^{\prime}$ \\
\hline & & & $\perp \mathrm{C}_{3 z} ;$ or a.o. & $\begin{array}{l}11^{\prime} \\
m_{r} 1^{\prime}\end{array}$ \\
\hline & & & $\mid \begin{array}{l}\| \sigma_{d i} \\
\perp \sigma_{d i}\end{array}$ & $m_{z} 1^{\prime}$ \\
\hline \multirow[t]{3}{*}{117} & $\overline{3} m 1^{\prime}$ & 82 & $\| \mathrm{C}_{\mathbf{3} z}$ & $\overline{3} m 1^{\prime}$ \\
\hline & & & $\begin{array}{l}\perp \mathrm{C}_{3 z} ; \text { or a.o. } \\
\| \mathrm{C}_{2 ;}^{\prime}\end{array}$ & $\begin{array}{l}11^{\prime} \\
2_{z} / m_{z} 1^{\prime}\end{array}$ \\
\hline & & & $\perp \mathrm{C}_{2 i}^{\prime}$ & $2_{r} / m_{r} 1^{\prime}$ \\
\hline \multirow[t]{4}{*}{118} & $231^{\prime}$ & 83 & $\| \mathrm{C}_{2 m}$ & $2221^{\prime}$ \\
\hline & & & $\perp \mathrm{C}_{2 m}$ & $21^{\prime}$ \\
\hline & & & $\| \mathrm{C}_{3 j}$ & $\begin{array}{l}31^{\prime} \\
11^{\prime}\end{array}$ \\
\hline & $m 31^{\prime}$ & 83 & $\begin{array}{l}\perp \mathrm{C}_{3 j} ; \text { or a.o. } \\
\| \mathrm{C}_{2 m}\end{array}$ & $\begin{array}{l}11^{\prime} \\
m m m 1^{\prime}\end{array}$ \\
\hline \multirow{3}{*}{119} & & & $\perp \mathrm{C}_{2 m}$ & $2_{r} / m_{r} 1^{\prime}$ \\
\hline & & & $\begin{array}{l}\| \mathrm{C}_{3 j} \\
\perp \mathrm{C}_{3 i} ; \text { or a.o. }\end{array}$ & $\begin{array}{l}31^{\prime} \\
11^{\prime}\end{array}$ \\
\hline & & & & \\
\hline
\end{tabular}


TABLE I cont.

\begin{tabular}{|c|c|c|c|c|}
\hline (1) & (2) & $\overline{\overline{(3)}}$ & (4) & $\overline{(5)}$ \\
\hline \multirow[t]{5}{*}{120} & $4321^{\prime}$ & 83 & $\| \mathrm{C}_{4 m}$ & $4221^{\prime}$ \\
\hline & & & $\perp \mathrm{C}_{4 m}, \mathrm{C}_{2 p}$ & $2_{r} 1^{\prime}$ \\
\hline & & & $\| \mathrm{C}_{2 p}$ & $2221^{\prime}$ \\
\hline & & & $\| C_{3 j}$ & $321^{\prime}$ \\
\hline & & & $\perp C_{3 j}$; or a.o. & $11^{\prime}$ \\
\hline \multirow[t]{5}{*}{121} & $\overline{4} 3 m 1^{\prime}$ & 83 & $\| S_{4 m}$ & $\overline{4} 2 m 1^{\prime}$ \\
\hline & & & $\perp \mathbf{S}_{4 m}$ & $21^{\prime}$ \\
\hline & & & $\| \mathrm{C}_{3 j}$ & $3 m 1^{\prime}$ \\
\hline & & & $\begin{array}{l}\perp C_{3 j} ; \text { or a.o. } \\
\perp \sigma_{d p}\end{array}$ & $\begin{array}{l}11^{\prime} \\
m 2 m 1^{\prime}, 2 m m 1^{\prime}\end{array}$ \\
\hline & & & $\| \sigma_{d p}$ & $m_{r} 1^{\prime}$ \\
\hline \multirow[t]{4}{*}{122} & $m 3 m 1^{\prime}$ & 83 & $\| \mathrm{C}_{4 m}$ & $4 / m m m 1^{\prime}$ \\
\hline & & & $\begin{array}{l}1 C_{4 m}, C_{2 p} \\
\| C_{2 n}\end{array}$ & $\begin{array}{l}2_{r} / m_{r} 1^{\prime} \\
m m m 1^{\prime}\end{array}$ \\
\hline & & & $\| \mathrm{C}_{3 j}$ & $\overline{3} m 1^{\prime}$ \\
\hline & & & $\perp C_{3 j}$; or & $\overline{1} 1^{\prime}$ \\
\hline
\end{tabular}


TABLE II

The explicit form of the broken groups $\mathrm{K}(\boldsymbol{F})=\mathrm{J}(\boldsymbol{F})+b(\boldsymbol{F}) \mathrm{J}(\boldsymbol{F})$ for $\boldsymbol{F}=\boldsymbol{E}, \boldsymbol{k}, \boldsymbol{H}$, where $\mathrm{J}(\boldsymbol{F})$ contains the elements preserving field direction $\boldsymbol{F}, \boldsymbol{F} \rightarrow \boldsymbol{F}$, and $\boldsymbol{b}(\boldsymbol{F}) \mathrm{J}(\boldsymbol{F})$ reversing the field $\boldsymbol{F}$ to opposite, $\boldsymbol{F} \rightarrow-\boldsymbol{F}$.

For each group $K(\boldsymbol{F})$ the forms of the tensors $\varepsilon_{\mathrm{ij}}^{\mathrm{e}}$ and $\varepsilon_{\mathrm{ij}}^{\mathrm{o}}$ are also given (via table III).

\begin{tabular}{|c|c|c|c|c|c|c|c|c|c|}
\hline $\begin{array}{l}(1) \\
\mathrm{K}(F)\end{array}$ & $\begin{array}{l}(2) \\
\mathrm{J}(\boldsymbol{E})\end{array}$ & $\begin{array}{l}(3) \\
b(E)\end{array}$ & $\begin{array}{l}\overline{(4)} \\
\varepsilon_{\mathrm{ij}}\end{array}$ & $\begin{array}{l}(5) \\
\mathrm{J}(k)\end{array}$ & $\begin{array}{l}(6) \\
b(k)\end{array}$ & $\begin{array}{l}(7) \\
\varepsilon_{\mathrm{ij}}\end{array}$ & $\begin{array}{l}(8) \\
\mathrm{J}(H)\end{array}$ & $\begin{array}{l}(9) \\
b(\boldsymbol{H})\end{array}$ & $\begin{array}{l}(10) \\
\varepsilon_{\mathrm{ij}}\end{array}$ \\
\hline 1 & 1 & - & 1 & 1 & - & 1 & 1 & - & 1 \\
\hline$\overline{1}$ & 1 & I & 18 & 1 & I & 18 & $\overline{1}$ & - & 1 \\
\hline $2_{x}$ & 1 & $\mathrm{C}_{2 x}$ & 19 & 1 & $\mathrm{C}_{2 x}$ & 19 & 1 & $\mathrm{C}_{2 x}$ & 19 \\
\hline $2_{y}$ & 1 & $\mathrm{C}_{2 y}$ & 20 & 1 & $\mathrm{C}_{2 y}$ & 20 & 1 & $\mathrm{C}_{2 y}$ & 20 \\
\hline $2 z$ & $2 z_{z}$ & - & 2 & $2 z_{z}$ & - & 2 & $2_{z}$ & - & 2 \\
\hline$m_{x}$ & $m_{x}$ & - & 3 & $m_{x}$ & - & 3 & 1 & $\sigma_{x}$ & 19 \\
\hline$m_{y}$ & $m_{y}$ & - & 4 & $m_{y}$ & - & 4 & 1 & $\sigma_{y}$ & 20 \\
\hline$m_{z}$ & 1 & $\sigma_{z}$ & 21 & 1 & $\sigma_{z}$ & 21 & $m_{z}$ & - & 2 \\
\hline $2_{x} / m_{x}$ & $m_{x}$ & I & 22 & $m_{x}$ & I & 22 & $\overline{1}$ & $\mathrm{C}_{2 x}$ & 19 \\
\hline $2_{y} / m_{y}$ & $m_{y}$ & I & 23 & $m_{y}$ & I & 23 & $\overline{1}$ & $\mathrm{C}_{2 y}$ & 20 \\
\hline $2_{z} / m_{z}$ & $2_{z}$ & I & 24 & $2 z$ & I & 24 & $2_{z} / m_{z}$ & - & 2 \\
\hline 222 & $2_{z}$ & $\mathrm{C}_{2 x}$ & 25 & $2 z$ & $\mathrm{C}_{2 x}$ & 25 & $2 z_{z}$ & $\mathrm{C}_{2 x}$ & 25 \\
\hline$m m 2$ & $m m 2$ & - & 5 & $m m 2$ & - & 5 & $2 z$ & $\sigma_{x}$ & 25 \\
\hline$m 2 m$ & $m_{x}$ & $\sigma_{z}$ & 26 & $m_{x}$ & $\sigma_{z}$ & 26 & $m_{z}$ & $\mathrm{C}_{2 y}$ & 25 \\
\hline $2 \mathrm{~mm}$ & $m_{y}$ & $\sigma_{z}$ & 27 & $m_{y}$ & $\sigma_{z}$ & 27 & & $\mathrm{C}_{2 x}$ & 25 \\
\hline$m m m$ & $m m 2$ & I & 28 & $m m 2$ & I & 28 & $2_{z} / m_{z}$ & $\mathrm{C}_{2 x}$ & 25 \\
\hline 3 & 3 & - & 6 & 3 & - & 6 & 3 & - & 6 \\
\hline$\overline{3}$ & 3 & I & 29 & 3 & I & 29 & $\overline{3}$ & - & 6 \\
\hline 32 & 3 & $\mathrm{C}_{2 x}$ & 30 & 3 & $\mathrm{C}_{2 x}$ & 30 & 3 & $\mathrm{C}_{2 x}$ & 30 \\
\hline $3 m$ & $3 m$ & - & 7 & $3 m$ & - & 7 & 3 & $\sigma_{x}$ & 30 \\
\hline$\overline{3} m$ & $3 m$ & I & 31 & $3 m$ & I & 31 & $\overline{3}$ & $\sigma_{x}$ & 30 \\
\hline 4 & 4 & - & 6 & 4 & - & 6 & 4 & - & 6 \\
\hline$\overline{4}$ & $2 z$ & $\mathrm{~S}_{4 z}$ & 32 & $2 z$ & $S_{4 z}$ & 32 & $\overline{4}$ & - & 6 \\
\hline $4 / m$ & 4 & I & 29 & 4 & I & 29 & $4 / m$ & - & 6 \\
\hline 422 & 4 & $\mathrm{C}_{2 x}$ & 30 & 4 & $\mathrm{C}_{2 x}$ & 30 & 4 & $\mathrm{C}_{2 x}$ & 30 \\
\hline $4 m m$ & $4 m m$ & - & 7 & $4 m m$ & - & 7 & 4 & $\sigma_{x}$ & 30 \\
\hline$\overline{4} 2 m$ & $m m 2$ & $S_{4 z}$ & 33 & $m m 2$ & $\mathrm{~S}_{4 z}$ & 33 & $\overline{4}$ & $\mathrm{C}_{2 x}$ & 30 \\
\hline $4 / m m m$ & $4 m m$ & I & 31 & $4 m m$ & I & 31 & $4 / m$ & $\mathrm{C}_{2 x}$ & 30 \\
\hline 6 & 6 & - & 6 & 6 & - & 6 & 6 & - & 6 \\
\hline$\overline{6}$ & 3 & $\sigma_{z}$ & 29 & 3 & $\sigma_{z}$ & 29 & $\overline{6}$ & - & 6 \\
\hline $6 / m$ & 6 & I & 29 & 6 & I & 29 & $6 / m$ & - & 6 \\
\hline 622 & 6 & $\mathrm{C}_{2 x}$ & 30 & 6 & $\mathrm{C}_{2 x}$ & 30 & 6 & $\mathrm{C}_{2 x}$ & 30 \\
\hline $6 m m$ & $6 m m$ & - & 7 & $6 \mathrm{~mm}$ & - & 7 & 6 & $\sigma_{x}$ & 30 \\
\hline$\overline{6} 2 m$ & $3 m$ & $\sigma_{z}$ & 31 & $3 m$ & $\mathrm{C}_{2 x}$ & 31 & $\overline{6}$ & $\mathrm{C}_{2 x}$ & 30 \\
\hline $6 / \mathrm{mmm}$ & $6 \mathrm{~mm}$ & I & 31 & $6 m m$ & I & 31 & $6 / m$ & $\mathrm{C}_{2 x}$ & 30 \\
\hline & & - & 8 & & $\theta$ & 41 & & $\Theta$ & 41 \\
\hline
\end{tabular}


TABLE II cont.

\begin{tabular}{|c|c|c|c|c|c|c|c|c|c|}
\hline (1) & $(2)$ & (3) & (4) & $\overline{(5)}$ & $\overline{(6)}$ & 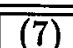 & $\overline{(18)}$ & $\overline{(\overline{(9)}}$ & 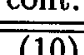 \\
\hline$\overline{1} 1^{\prime}$ & $11^{\prime}$ & $\mathrm{I}$ & 40 & $\overrightarrow{1}$ & $\theta$ & 40 & $\overline{1}$ & $\theta$ & $\frac{(10)}{41}$ \\
\hline $2{ }_{x} 1^{\prime}$ & $11^{\prime}$ & $\mathrm{C}_{2 x}$ & 42 & $2_{x}^{\prime}$ & $\Theta$ & 54 & $2^{\prime}$ & $\Theta$ & $\begin{array}{l}41 \\
54\end{array}$ \\
\hline $21^{\prime}$ & $11^{\prime}$ & $\mathrm{C}_{2 y}$ & 43 & $2_{y}^{\prime}$ & $\Theta$ & 55 & $2_{y}^{\prime x}$ & $\Theta$ & 55 \\
\hline $2 z 1^{\prime}$ & $2_{z}^{\prime}$ & - & 9 & $2 y$ & $\theta$ & 56 & $2{ }_{z}$ & $\Theta$ & 56 \\
\hline$m_{x} 1^{\prime}$ & $m_{x} 1^{\prime}$ & - & 10 & $m_{x}$ & $\theta$ & 57 & $m_{x}^{\prime}$ & $\Theta$ & 54 \\
\hline$m_{y} 1^{\prime}$ & $m_{y} 1^{\prime}$ & - & 11 & $m_{y}$ & $\theta$ & 58 & $m_{y}^{\prime}$ & $\Theta$ & 55 \\
\hline$m_{z} 1^{\prime}$ & $11^{\prime}$ & $\sigma_{z}$ & 44 & $m_{z}^{\prime}$ & $\theta$ & 59 & $m_{z}^{y}$ & $\Theta$ & 56 \\
\hline $2_{x} / m_{x} 1^{\prime}$ & $m_{x} 1^{\prime}$ & I & 47 & $2_{x}^{\prime} / m_{x}$ & $\theta$ & 47 & $2_{x}^{\prime} / m_{x}^{\prime}$ & $\Theta$ & 54 \\
\hline $2_{y} / m_{y}^{\prime} 1^{\prime}$ & $m_{y} 1^{\prime}$ & I & 46 & $2_{y}^{\prime} / m_{y}$ & $\theta$ & 46 & $2_{u}^{\prime} / m_{v}^{\prime}$ & $\theta$ & 55 \\
\hline $2_{z} / m_{z} 1^{\prime}$ & $2 z 1^{\prime}$ & I & 45 & $22_{z} / m_{z}^{\prime}$ & $\theta$ & 45 & $2_{z} / m_{z}$ & $\Theta$ & 56 \\
\hline $2221^{\prime}$ & $2_{z} 1^{\prime}$ & $\mathrm{C}_{2 x}$ & 48 & $2^{\prime} 2^{\prime} 2$ & $\theta$ & 72 & $2^{\prime} 2^{\prime} 2$ & $\theta$ & 72 \\
\hline$m m 21^{\prime}$ & $m m 21^{\prime}$ & - & 5 & $m m 2$ & $\Theta$ & 28 & $m^{\prime} m^{\prime} 2$ & $\Theta$ & 72 \\
\hline$m 2 m 1^{\prime}$ & $m_{x} 1^{\prime}$ & $\mathrm{C}_{2 y}$ & 49 & $m 2^{\prime} m^{\prime}$ & $\Theta$ & 73 & $m^{\prime} 2^{\prime} m$ & $\Theta$ & 72 \\
\hline $2 m m 1^{\prime}$ & $m_{y} 1^{\prime}$ & $\mathrm{C}_{2 x}$ & 50 & $2^{\prime} m m^{\prime}$ & $\Theta$ & 74 & $2^{\prime} m^{\prime} m$ & $\Theta$ & 72 \\
\hline$m m m 1^{\prime}$ & $m m 21^{\prime}$ & I & 28 & $m m m^{\prime}$ & $\Theta$ & 28 & $m^{\prime} m^{\prime} m$ & $\Theta$ & 72 \\
\hline $31^{\prime}$ & $31^{\prime}$ & - & 7 & 3 & $\Theta$ & 30 & 3 & $\Theta$ & 30 \\
\hline$\overline{3} 1^{\prime}$ & $31^{\prime}$ & I & 31 & $\overline{3}^{\prime}$ & $\Theta$ & 31 & $\overline{3}$ & $\Theta$ & 30 \\
\hline $321^{\prime}$ & $31^{\prime}$ & $\mathrm{C}_{2 x}$ & 31 & $32^{\prime}$ & $\Theta$ & 30 & $32^{\prime}$ & $\Theta$ & 30 \\
\hline $3 m 1^{\prime}$ & $3 m 1^{\prime}$ & - & 7 & $3 m$ & $\Theta$ & 31 & $3 m^{\prime}$ & $\Theta$ & 30 \\
\hline$\overline{3} m 1^{\prime}$ & $3 m 1^{\prime}$ & I & 31 & $\overrightarrow{3} m$ & $\Theta$ & 31 & $\overline{3} m^{\prime}$ & $\Theta$ & 30 \\
\hline $41^{\prime}$ & $41^{\prime}$ & - & 7 & 4 & $\Theta$ & 30 & 4 & $\Theta$ & 30 \\
\hline$\overline{4} 1^{\prime}$ & $2_{z} 1^{\prime}$ & $\mathrm{S}_{4 z}$ & 34 & $\overrightarrow{4}$ & $\Theta$ & 31 & $\overline{4}$ & $\Theta$ & 30 \\
\hline $4 / m 1^{\prime}$ & $41^{\prime}$ & I & 31 & $4 / m^{\prime}$ & $\Theta$ & 31 & $4 / m$ & $\theta$ & 30 \\
\hline $4221^{\prime}$ & $41^{\prime}$ & $\mathrm{C}_{2 x}$ & 31 & $42^{\prime} 2^{\prime}$ & $\Theta$ & 30 & $42^{\prime} 2^{\prime}$ & $\Theta$ & 30 \\
\hline $4 m m 1^{\prime}$ & $4 m m 1^{\prime}$ & - & 7 & $4 \mathrm{~mm}$ & $\Theta$ & 31 & $4 m^{\prime} m^{\prime}$ & $\Theta$ & 30 \\
\hline$\overline{4} 2 m 1^{\prime}$ & $m m 21^{\prime}$ & $\mathrm{S}_{4 z}$ & 33 & $\overline{4}^{\prime} 2^{\prime} m$ & $\Theta$ & 31 & $\overline{4} 2^{\prime} m^{\prime}$ & $\Theta$ & 30 \\
\hline $4 / m m m 1^{\prime}$ & $4 m m 1^{\prime}$ & I & 31 & $4 / m^{\prime} m m$ & $\Theta$ & 31 & $4 / m m^{\prime} m^{\prime}$ & $\Theta$ & 30 \\
\hline $61^{\prime}$ & $61^{\prime}$ & - & 7 & 6 & $\Theta$ & 30 & 6 & $\theta$ & 30 \\
\hline$\overline{6} 1^{\prime}$ & $31^{\prime}$ & $\sigma_{z}$ & 31 & $\overline{6}^{\prime}$ & $\Theta$ & 31 & $\overline{6}$ & $\theta$ & 30 \\
\hline $6 / m 1^{\prime}$ & $61^{\prime}$ & I & 31 & $6 / m^{\prime}$ & $\Theta$ & 31 & $6 / m$ & $\theta$ & 30 \\
\hline $6221^{\prime}$ & & $\mathrm{C}_{2 x}$ & 31 & $62^{\prime} 2^{\prime}$ & $\Theta$ & 30 & $62^{\prime} 2^{\prime}$ & $\theta$ & 30 \\
\hline $6 m m 1^{\prime}$ & $6 m m 1^{\prime}$ & - & 7 & $6 \mathrm{~mm}$ & $\theta$ & 31 & $6 m^{\prime} m^{\prime}$ & $\theta$ & 30 \\
\hline$\overline{6} 2 m 1^{\prime}$ & $3 m 1^{\prime}$ & $\sigma_{z}$ & 31 & $\overline{6}^{\prime} 2^{\prime} m$ & $\Theta$ & 31 & $\overline{6} 2^{\prime} m^{\prime}$ & $\Theta$ & 30 \\
\hline $6 / m m m 1^{\prime}$ & $6 m m 1^{\prime}$ & I & 31 & $6 / m^{\prime} m m$ & $\Theta$ & 31 & $6 / m m^{\prime} m^{\prime}$ & $\theta$ & 30 \\
\hline$\overline{1}^{\prime}$ & 1 & $I^{\prime}$ & 41 & $\overrightarrow{1}$ & - & 8 & 1 & $\mathbf{I}^{\prime}$ & 41 \\
\hline $2_{x}^{\prime}$ & 1 & $\mathrm{C}_{2 x}^{\prime}$ & 51 & $2_{x}^{\prime}$ & - & 13 & $2_{x}^{\prime}$ & - & 13 \\
\hline $22_{y}^{\prime \prime}$ & 1 & $\mathrm{C}_{2 y}^{\prime}$ & 52 & $22_{y}^{\prime}$ & - & 14 & $2_{y}^{\prime \prime}$ & - & 14 \\
\hline $2_{z}^{\prime}$ & $2_{z}^{\prime}$ & - & 12 & 1 & $\mathrm{C}_{2 z}^{\prime}$ & 53 & 1 & $\mathrm{C}_{2 z}^{\prime}$ & 53 \\
\hline$m_{x}^{\prime}$ & $m_{x}^{\prime}$ & - & 13 & 1 & $\sigma_{x}^{\prime}$ & 51 & $m_{x}^{\prime}$ & - & 13 \\
\hline$m_{y}^{\prime}$ & $m_{y}^{\prime}$ & - & 14 & 1 & $\sigma_{y}^{i}$ & 52 & $m_{y}^{\prime}$ & - & 14 \\
\hline
\end{tabular}


TABLE II cont.

\begin{tabular}{|c|c|c|c|c|c|c|c|c|c|}
\hline (1) & (2) & (3) & (4) & (5) & $(6)$ & (7) & (8) & (9) & $(10)$ \\
\hline$m_{z}^{\prime}$ & 1 & $\sigma_{z}^{\prime}$ & 53 & $m_{z}^{\prime}$ & - & 12 & 1 & $\sigma_{z}^{\prime}$ & 53 \\
\hline $2_{x} / m_{x}^{\prime}$ & $m_{x}^{\prime}$ & $\mathrm{C}_{2 x}$ & 54 & $\overrightarrow{1}^{2}$ & $\mathrm{C}_{2 x}$ & 42 & $m_{x}^{\prime}$ & $\mathbf{1}^{\prime}$ & 54 \\
\hline $2_{y} / m_{y}^{\prime}$ & $m_{y}^{\prime}$ & $\mathrm{C}_{2 y}$ & 55 & $\overrightarrow{1}$ & $\mathrm{C}_{2 y}$ & 43 & $m_{y}^{\prime}$ & $\mathrm{I}^{\prime}$ & 55 \\
\hline $2_{z} / m_{z}^{\prime}$ & $2 z$ & $\sigma_{z}^{\prime}$ & 56 & $2_{z} / m_{z}^{\prime}$ & - & 9 & $2 z$ & $\mathrm{I}^{\prime}$ & 56 \\
\hline $2_{x}^{\prime} / m_{x}$ & $m_{x}$ & $\mathrm{C}_{2 x}^{\prime}$ & 57 & $2_{x}^{\prime} / m_{z}$ & - & 10 & $2_{x}^{\prime}$ & $\mathbf{I}^{\prime}$ & 54 \\
\hline $2_{y}^{\prime} / m_{y}$ & $m_{y}$ & $\mathrm{C}_{2 y}^{\prime}$ & 58 & $2_{y}^{\prime} / m_{y}$ & - & 11 & $2_{y}^{\prime}$ & $\mathrm{I}^{\prime}$ & 55 \\
\hline $2_{z}^{\prime} / m_{z}$ & $2_{z}^{\prime}$ & $\sigma_{z}$ & 59 & $\overrightarrow{1}$ & $\sigma_{z}$ & 44 & $m_{z}$ & $I^{\prime}$ & 56 \\
\hline $2_{x}^{\prime} / m_{x}^{\prime}$ & $m_{x}^{\prime}$ & I & 60 & $2_{x}^{\prime}$ & $\mathrm{I}$ & 60 & $2_{x}^{\prime} / m_{x}^{\prime}$ & - & 13 \\
\hline $2_{x}^{\prime} / m_{y}^{\prime}$ & $m_{y}^{\prime}$ & I & 61 & $2_{y}^{\prime}$ & I & 61 & $2_{y}^{\prime} / m_{y}^{\prime}$ & - & 14 \\
\hline $2_{z}^{\prime} / m_{z}^{\prime}$ & $2_{z}^{\prime}$ & I & 62 & $m_{z}^{\prime}$ & I & 62 & & $\mathrm{C}_{2 z}^{\prime}$ & 53 \\
\hline $2^{\prime} 2^{\prime} 2$ & $2 z$ & $\mathrm{C}_{2 x}^{\prime}$ & 63 & $2^{\prime} 2^{\prime} 2$ & - & 15 & $2^{\prime} 2^{\prime} 2$ & - & 15 \\
\hline $2^{\prime} 22^{\prime}$ & $2_{z}^{\prime}$ & $\mathrm{C}_{2 y}$ & 64 & $2_{x}^{\prime}$ & $\mathrm{C}_{2 z}^{\prime}$ & 66 & $2_{x}^{\prime}$ & $\mathrm{C}_{2 z}^{\prime}$ & 66 \\
\hline $22^{\prime} 2^{\prime}$ & & $\mathrm{C}_{2 x}$ & 65 & $2_{y}^{\prime}$ & $\mathrm{C}_{2 z}^{\prime}$ & 67 & $2_{y}^{\prime}$ & $\mathrm{C}_{2 z}^{\prime}$ & 67 \\
\hline$m^{\prime} m^{\prime} 2$ & $m^{\prime} m^{\prime} 2$ & - & 15 & $2 z$ & $\sigma_{x}^{\prime}$ & 63 & $m^{\prime} m^{\prime} 2$ & - & 15 \\
\hline$m^{\prime} 2 m^{\prime}$ & $m_{x}^{\prime}$ & $\mathrm{C}_{2 y}$ & 66 & $m_{z}^{\prime}$ & $\mathrm{C}_{2 y}$ & 64 & $m_{x}^{\prime}$ & $\sigma_{z}^{\prime}$ & 66 \\
\hline $2 m^{\prime} m^{\prime}$ & $m_{y}^{\prime}$ & $\mathrm{C}_{2 x}$ & 67 & $m_{z}^{\prime}$ & $\mathrm{C}_{2 x}$ & 65 & $m_{y}^{\prime}$ & $\sigma_{z}^{\prime}$ & 67 \\
\hline$m^{\prime} 2^{\prime} m$ & $m_{x}^{\prime}$ & $\sigma_{z}$ & 68 & $2_{y}^{\prime \prime}$ & $\sigma_{z}$ & 69 & $m^{\prime} 2^{\prime} m$ & - & 15 \\
\hline $2^{\prime} m^{\prime} m$ & $m_{y}^{\prime}$ & $\sigma_{z}$ & 69 & $2_{x}^{\prime}$ & $\sigma_{z}$ & 68 & $2^{\prime} m^{\prime} m$ & - & 15 \\
\hline$m^{\prime} m 2^{\prime}$ & $m^{\prime} m 2^{\prime}$ & - & 16 & $m_{y}$ & $\mathrm{C}_{2 z}^{\prime}$ & 70 & $m_{x}^{\prime}$ & $\mathrm{C}_{2 z}^{\prime}$ & 66 \\
\hline $2^{\prime} m m^{\prime}$ & $m_{y}$ & $\sigma_{z}^{\prime}$ & 70 & $2^{\prime} m m^{\prime}$ & - & 16 & $2_{x}^{\prime}$ & $\sigma_{z}^{\prime}$ & 66 \\
\hline$m 2^{\prime} m^{\prime}$ & $m_{x}$ & $\sigma_{z}^{\prime}$ & 71 & $m 2^{\prime} m^{\prime}$ & - & 17 & $2_{y}^{\prime}$ & $\sigma_{z}^{\prime}$ & 67 \\
\hline$m m^{\prime} 2^{\prime}$ & $m m^{\prime} 2^{\prime}$ & - & 17 & $m_{x}$ & $\mathrm{C}_{2 z}^{\prime}$ & 71 & $m_{y}^{\prime}$ & $\mathrm{C}_{2 z}^{\prime}$ & 67 \\
\hline$m^{\prime} m^{\prime} m^{\prime}$ & $m^{\prime} m^{\prime} 2$ & $\mathrm{I}^{\prime}$ & 72 & $2_{z} / m_{z}^{\prime}$ & $\mathrm{C}_{2 x}$ & 48 & $m^{\prime} m^{\prime} 2$ & $\mathrm{I}^{\prime}$ & 72 \\
\hline$m m m^{\prime}$ & $m m 2$ & $\mathbf{I}^{\prime}$ & 28 & $m m m^{\prime}$ & - & 5 & $2^{\prime} 2^{\prime} 2$ & $\mathbf{I}^{\prime}$ & 72 \\
\hline$m m^{\prime} m$ & $m m^{\prime} 2^{\prime}$ & $\mathbf{I}^{\prime}$ & 73 & $2_{x}^{\prime} / m_{x}$ & $\mathrm{C}_{2 y}$ & 49 & $2^{\prime} m^{\prime} m$ & $\mathbf{I}^{\prime}$ & 72 \\
\hline$m^{\prime} m m$ & $m^{\prime} m 2^{\prime}$ & $\mathrm{I}^{\prime}$ & 74 & $2_{y}^{\prime} / m_{y}$ & $\mathrm{C}_{2 x}$ & 50 & $m^{\prime} 2^{\prime} m$ & $I^{\prime}$ & 72 \\
\hline$m^{\prime} m^{\prime} m$ & $m^{\prime} m^{\prime} 2$ & I & 75 & $2^{\prime} 2^{\prime} 2$ & I. & 75 & $m^{\prime} m^{\prime} m$ & - & 15 \\
\hline$m^{\prime} m m^{\prime}$ & $m^{\prime} m 2^{\prime}$ & I & 76 & $2^{\prime} m m^{\prime}$ & I & 76 & $2_{x}^{\prime} / m_{x}^{\prime}$ & $\mathrm{C}_{2 z}^{\prime}$ & 66 \\
\hline$m m^{\prime} m^{\prime}$ & $m m^{\prime} 2^{\prime}$ & I & 77 & $m 2^{\prime} m^{\prime}$ & I & 77 & $2_{y}^{\prime} / m_{y}^{\prime}$ & $\mathrm{C}_{2 z}^{\prime}$ & 67 \\
\hline$\overline{3}^{\prime}$ & 3 & $\mathbf{I}^{\prime}$ & 30 & $\overrightarrow{3}$ & - & 7 & 3 & $\mathbf{I}^{\prime}$ & 30 \\
\hline $32^{\prime}$ & 3 & $\mathrm{C}_{2 x}^{\prime}$ & 29 & $32^{\prime}$ & - & 6 & $32^{\prime}$ & - & 6 \\
\hline $3 m^{\prime}$ & $3 m^{\prime}$ & - & 6 & 3 & $\sigma_{x}^{\prime}$ & 29 & $3 m^{\prime}$ & - & 6 \\
\hline$\overline{3} m^{\prime}$ & $3 m^{\prime}$ & I & 29 & $32^{\prime}$ & I & 29 & $\overline{3} m^{\prime}$ & - & 6 \\
\hline$\overline{3}^{\prime} m$ & $3 m$ & $\mathrm{I}^{\prime}$ & 31 & $\overline{3}^{\prime} m$ & - & 7 & $32^{\prime}$ & $\mathbf{I}^{\prime}$ & 30 \\
\hline$\overline{3}^{\prime} m^{\prime}$ & $3 m^{\prime}$ & $\mathbf{1}^{\prime}$ & 30 & $\overline{3}^{\prime}$ & $\sigma_{x}^{\prime}$ & 31 & $3 m^{\prime}$ & $\mathrm{I}^{\prime}$ & 30 \\
\hline $4^{\prime}$ & $4^{\prime}$ & - & 7 & $2_{z}$ & $\mathrm{C}_{4 z}^{\prime}$ & 35 & $2_{z}$ & $\mathrm{C}_{4 z}^{\prime}$ & 35 \\
\hline$\overline{4}^{\prime}$ & $2 z$ & $\mathbf{S}_{4 z}^{\prime}$ & 35 & & - & 7 & $2 z$ & $S_{4 z}^{\prime}$ & 35 \\
\hline $4 / m^{\prime}$ & 4 & $\mathbf{I}^{\prime}$ & 30 & $4 / m^{\prime}$ & - & 7 & 4 & $\mathbf{I}^{\prime}$ & 30 \\
\hline
\end{tabular}


TABLE II cont.

\begin{tabular}{|c|c|c|c|c|c|c|c|c|c|}
\hline (1) & (2) & $(3)$ & $\overline{(4)}$ & $\overline{(5)}$ & (6) & $\overline{(7)}$ & $\overline{(8)}$ & $\overline{(9)}$ & $\overline{(10)}$ \\
\hline $4^{\prime} / m$ & $4^{\prime}$ & I & 31 & $\overrightarrow{4}^{\prime}$ & I & 31 & $\underline{2} / m_{z}$ & $\mathrm{C}_{4 z}^{\prime}$ & 35 \\
\hline $4^{\prime} / m^{\prime}$ & $4^{\prime}$ & $I^{\prime}$ & 31 & $2_{z} / m_{z}^{\prime}$ & $\mathrm{C}_{4 z}^{\prime}$ & 34 & & $\mathrm{I}^{\prime 2 z}$ & 30 \\
\hline $42^{\prime} 2^{\prime}$ & 4 & $\mathrm{C}_{2 x}^{\prime}$ & 29 & $42^{\prime} 2^{\prime}$ & $-4 z$ & 6 & $42^{\prime} 2^{\prime}$ & - & 6 \\
\hline $4^{\prime} 22^{\prime}$ & $4^{\prime}$ & $\mathrm{C}_{2 x}$ & 31 & $2^{\prime} 2^{\prime} 2$ & $\mathrm{C}_{4 z}^{\prime}$ & 36 & $2^{\prime} 2^{\prime} 2$ & $\mathrm{C}_{4 z}^{\prime}$ & 36 \\
\hline $4 m^{\prime} m^{\prime}$ & $4 m^{\prime} m^{\prime}$ & - & 6 & 4 & $\sigma_{x}^{\prime z}$ & 29 & $4 m^{\prime} m^{\prime}$ & - & 6 \\
\hline $4^{\prime} m m^{\prime}$ & $4^{\prime} m m^{\prime}$ & - & 7 & $m m 2$ & $\mathrm{C}_{4 z}^{\prime}$ & 38 & $m_{a}^{\prime} m_{b}^{\prime} 2$ & $\mathrm{C}_{4 z}^{\prime}$ & 36 \\
\hline$\overline{4} 2^{\prime} m^{\prime}$ & $m^{\prime} m^{\prime} 2$ & $\mathrm{~S}_{4 z}$ & 37 & $2^{\prime} 2^{\prime} 2$ & $\mathrm{~S}_{4 z}$ & 39 & $\overline{4} 2^{\prime} m^{\prime}$ & $-\infty$ & 6 \\
\hline$\overline{4}^{\prime} 2 m^{\prime}$ & $m^{\prime} m^{\prime} 2$ & $\mathrm{~S}_{4 z}^{\prime}$ & 36 & $\overline{4}^{\prime}$ & $\mathrm{C}_{2 x}$ & 31 & $m_{a}^{\prime} m_{b}^{\prime} 2$ & $S_{4 z}^{\prime}$ & 36 \\
\hline$\overline{4}^{\prime} m 2^{\prime}$ & $m m 2$ & $S_{4 z}^{\prime}$ & 38 & $\overline{4}^{\prime} m 2^{\prime}$ & - & 7 & $2_{a}^{\prime} 2_{b}^{\prime} 2$ & $S_{4 z}^{\prime}$ & 36 \\
\hline $4 / m^{\prime} m^{\prime} m^{\prime}$ & $4 m^{\prime} m^{\prime}$ & $\mathrm{I}^{\prime}$ & 30 & $4 / m^{\prime}$ & $\mathrm{C}_{2 x}$ & 31 & $4 m^{\prime} m^{\prime}$ & $\mathrm{I}^{\prime}$ & 30 \\
\hline $4 / m^{\prime} m m$ & $4 m m$ & $\mathbf{I}^{\prime}$ & 31 & $4 / m^{\prime} m m$ & - & 7 & $42^{\prime} 2^{\prime}$ & $\mathbf{I}^{\prime}$ & 30 \\
\hline $4^{\prime} / \mathrm{mmm}^{\prime}$ & $4^{\prime} m m^{\prime}$ & I & 31 & $\overline{4}^{i} m 2^{\prime}$ & I & 31 & $m_{a}^{\prime} m_{b}^{\prime} m$ & $\mathrm{C}_{4 z}^{\prime}$ & 36 \\
\hline $4^{\prime} / m^{\prime} m^{\prime} m$ & $4^{\prime} \mathrm{mm}^{\prime}$ & $\mathbf{I}^{\prime}$ & 31 & $m_{a} m_{b} m^{\prime}$ & $S_{4 z}$ & 33 & $\overline{4} 2^{\prime} m^{\prime}$ & $I^{\prime}$ & 30 \\
\hline $4 / m m^{\prime} m^{\prime}$ & $4 m^{\prime} m^{\prime}$ & I & 29 & $42^{\prime} 2^{\prime}$ & I & 29 & $4 / m m^{\prime} m^{\prime}$ & - & 6 \\
\hline $6^{\prime}$ & $6^{\prime}$ & - & 7 & 3 & $\mathrm{C}_{2 z}^{\prime}$ & 30 & 3 & $\mathrm{C}_{2 z}^{\prime}$ & 30 \\
\hline$\overline{6}^{\prime}$ & 3 & $\sigma_{z}^{\prime}$ & 30 & $\overline{6}^{\prime}$ & - & 7 & 3 & $\sigma_{z}^{\prime}$ & 30 \\
\hline $6 / m^{\prime}$ & 6 & $\mathrm{I}^{\prime}$ & 30 & $6 / m^{\prime}$ & - & 7 & 6 & $\mathrm{I}^{\prime}$ & 30 \\
\hline $6^{\prime} / m^{\prime}$ & $6^{\prime}$ & I & 31 & $\overline{6}^{\prime}$ & I & 31 & $\overline{3}$ & $\sigma_{z}^{\prime}$ & 30 \\
\hline $6^{\prime} / m$ & $6^{\prime}$ & $\mathrm{I}^{\prime}$ & 31 & $\overline{3}^{\prime}$ & $\sigma_{z}$ & 31 & $\overline{6}$ & $\mathrm{I}^{\prime}$ & 30 \\
\hline $62^{\prime} 2^{\prime}$ & 6 & $\mathrm{C}_{2 x}^{\prime}$ & 29 & $62^{\prime} 2^{\prime}$ & - & 6 & $62^{\prime} 2^{\prime}$ & - & 6 \\
\hline $6^{\prime} 22^{\prime}$ & & $\mathrm{C}_{2 x}$ & 31 & $32^{\prime}$ & $C_{2 z}^{\prime}$ & 30 & $32^{\prime}$ & $\mathrm{C}_{2 z}^{\prime}$ & 30 \\
\hline $6 m^{\prime} m^{\prime}$ & $6 m^{\prime} m^{\prime}$ & - & 6 & 6 & $\sigma_{x}^{\prime 2}$ & 29 & $6 m^{\prime} m^{\prime}$ & - & 6 \\
\hline $6^{\prime} m m^{\prime}$ & $6^{\prime} \mathrm{mm}^{\prime}$ & - & 7 & $3 m$ & $\mathrm{C}_{2 z}^{\prime \prime}$ & 31 & $3 m^{\prime}$ & $\mathrm{C}_{2 z}^{\prime}$ & 30 \\
\hline$\overline{6} 2^{\prime} m^{\prime}$ & $3 m^{\prime}$ & $\sigma_{z}$ & 29 & $32^{\prime}$ & $\sigma_{z}$ & 29 & $\overline{6} 2^{\prime} m^{\prime}$ & & 6 \\
\hline$\overline{6}^{\prime} m 2^{\prime}$ & $3 m$ & $\sigma_{z}^{\prime}$ & 31 & $\overline{6}^{\prime} m 2^{\prime}$ & - & 7 & $32^{\prime}$ & $\sigma_{z}^{\prime}$ & 30 \\
\hline$\overline{6}^{\prime} m^{\prime} 2$ & $3 m^{\prime}$ & $\sigma_{z}^{\prime}$ & 30 & $\overline{6}^{\prime}$ & $\mathrm{C}_{2 x}$ & 31 & $3 m^{\prime}$ & $\sigma_{z}^{\prime}$ & 30 \\
\hline $6 / m^{\prime} m^{\prime} m^{\prime}$ & $6 m^{\prime} m^{\prime}$ & $\mathrm{I}^{\prime}$ & 30 & $6 / m^{\prime}$ & $\mathrm{C}_{2 x}$ & 31 & $6 m^{\prime} m^{\prime}$ & $\mathrm{I}^{\prime}$ & 30 \\
\hline $6 / m^{\prime} m m$ & $6 m m$ & $\mathrm{I}^{\prime}$ & 31 & $6 / m^{\prime} m m$ & - & 7 & $62^{\prime} 2^{\prime}$ & $\mathrm{I}^{\prime}$ & 30 \\
\hline $6^{\prime} / \mathrm{mmm}^{\prime}$ & $6^{\prime} m m^{\prime}$ & $\mathbf{I}^{\prime}$ & 31 & $\overline{3}^{\prime} m$ & $\sigma_{z}$ & 31 & $\overline{6} 2^{\prime} m^{\prime}$ & $\mathbf{I}^{\prime}$ & 30 \\
\hline $6^{\prime} / m^{\prime} m^{\prime} m$ & $6^{\prime} \mathrm{mm}^{\prime}$ & I & 31 & $\overline{6}^{\prime} 2^{\prime} m$ & I & 31 & $\overline{3} m^{\prime}$ & $\mathrm{C}_{2 z}^{\prime}$ & 30 \\
\hline $6 / m m^{\prime} m^{\prime}$ & $6 m^{\prime} m^{\prime}$ & I & 29 & $62^{\prime} 2^{\prime}$ & I & 29 & $6 / m m^{\prime} m^{\prime}$ & & 6 \\
\hline
\end{tabular}


TABLE III

The forms of the even $\varepsilon_{i j}^{e}\left(\varepsilon_{i j}^{e}(F)=\varepsilon_{i j}^{e}(-F)\right)$ and odd

$\varepsilon_{i j}^{\circ}\left(\varepsilon_{i j}^{o}(F)=-\varepsilon_{i j}^{\circ}(-F)\right)$ parts of the tensor $\tilde{\varepsilon}_{i j}=\varepsilon_{i j}^{\mathrm{e}}+\varepsilon_{i j}^{\circ}+\mathrm{i}\left(\varepsilon_{i j}^{l e}+\varepsilon_{i j}^{i o}\right)$.

The forms of the imaginary parts $\varepsilon_{i j}^{\prime e}$ and $\varepsilon_{i j}^{\prime o}$ are exactly the same as $\varepsilon_{i j}^{\mathrm{e}}$ and $\varepsilon_{i j}^{o}$, respectively. The components $11,22,33,12,21,13,31,23,32$ are denoted by symbols a, b, c, d, e, f, g, h, i, respectively.

The numbers $78-88$ refer to the tensors $\tilde{\varepsilon}_{i j}=\varepsilon_{i j}+i \varepsilon_{i j}^{\prime}$ of the groups K.

\begin{tabular}{|c|c|c|c|c|c|c|c|c|c|c|c|c|c|c|c|c|c|}
\hline \multicolumn{2}{|r|}{$\varepsilon_{i j}^{\mathrm{e}}$} & & \multicolumn{3}{|c|}{$\overline{\varepsilon_{i j}^{o}}$} & \multicolumn{3}{|c|}{$\varepsilon_{i j}^{e}$} & \multicolumn{3}{|c|}{$\overline{\varepsilon_{i j}^{o}}$} & \multicolumn{3}{|c|}{$\overline{\varepsilon_{i j}^{e}}$} & \multicolumn{3}{|c|}{$\varepsilon_{i j}^{o}$} \\
\hline 1 & & & & & & 2 & & & & & & 3 & & & & & \\
\hline $\mathbf{a}$ & $\mathrm{d}$ & $\mathrm{f}$ & a & $\mathbf{d}$ & $\mathbf{f}$ & $\mathbf{a}$ & d & 0 & $\mathbf{a}$ & d & 0 & $\mathbf{a}$ & 0 & & a & $\mathbf{0}$ & \\
\hline $\mathbf{e}$ & $\mathbf{b}$ & $\mathbf{h}$ & e & $\mathbf{b}$ & $\mathbf{h}$ & $\mathbf{e}$ & b & & $\mathbf{e}$ & b & 0 & 0 & b & & 0 & b & h \\
\hline $\begin{array}{l}\mathrm{g} \\
\mathbf{4}\end{array}$ & $\mathbf{i}$ & $\mathbf{c}$ & $\mathbf{g}$ & $\mathbf{i}$ & c & $\begin{array}{l}0 \\
5\end{array}$ & 0 & c & 0 & 0 & & 6 & & & 0 & & \\
\hline $\mathbf{a}$ & 0 & $f$ & $\mathbf{a}$ & 0 & $f$ & $\mathbf{a}$ & 0 & 0 & $\mathbf{a}$ & 0 & 0 & $\mathbf{a}$ & d & 0 & $\mathbf{a}$ & d & \\
\hline 0 & b & 0 & 0 & $\mathbf{b}$ & 0 & 0 & b & 0 & 0 & b & 0 & $-d$ & $\mathbf{a}$ & & $-d$ & $\mathbf{a}$ & \\
\hline $\begin{array}{l}g \\
7\end{array}$ & 0 & c & g & 0 & c & $\begin{array}{l}0 \\
8\end{array}$ & 0 & c & 0 & 0 & c & $\begin{array}{l}0 \\
9\end{array}$ & 0 & & 0 & 0 & \\
\hline $\mathbf{a}$ & 0 & 0 & $\mathbf{a}$ & 0 & 0 & $\mathbf{a}$ & d & f & $\mathbf{a}$ & d & & $\mathbf{a}$ & d & & $\mathbf{a}$ & d & \\
\hline 0 & $\mathbf{a}$ & 0 & 0 & a & 0 & d & b & h & $\mathrm{d}$ & $\mathrm{b}$ & h & d & b & & d & & \\
\hline 0 & 0 & c & 0 & 0 & $c$ & $\mathbf{f}$ & $\mathrm{b}$ & & & & & & 0 & & 0 & 0 & \\
\hline 10 & & & & & & 11 & & & & & & 12 & & & & & \\
\hline $\mathbf{a}$ & 0 & 0 & $\mathbf{a}$ & 0 & 0 & a & 0 & f & $\mathbf{a}$ & 0 & $f$ & $\mathbf{a}$ & d & & $\mathbf{a}$ & d & \\
\hline 0 & $\mathbf{b}$ & $\mathbf{h}$ & 0 & b & $\mathbf{h}$ & & $\mathrm{b}$ & 0 & & & & d & D & & $\mathrm{d}$ & b & h \\
\hline 0 & $\mathrm{~h}$ & c & 0 & $\mathbf{h}$ & $c$ & 1 & 0 & c & & & & [ & $-h$ & & $-f$ & $-h$ & \\
\hline 13 & & & & & & 14 & & & & & & 15 & & & & & \\
\hline $\mathbf{a}$ & d & f & $\mathbf{a}$ & $\mathrm{d}$ & $\mathbf{f}$ & 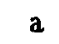 & $\mathrm{d}$ & f & $\mathbf{a}$ & $\mathrm{d}$ & & $\mathbf{a}$ & d & 0 & $\mathrm{a}$ & d & \\
\hline$-d$ & b & $\mathbf{h}$ & $-d$ & $\mathbf{b}$ & $\mathbf{h}$ & $-d$ & $\mathrm{~b}$ & $\mathbf{h}$ & $-d$ & b & $\mathrm{h}$ & $-d$ & 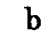 & 0 & $-d$ & b & v \\
\hline$-f$ & $\mathbf{h}$ & c & $-f$ & $\mathbf{h}$ & c & f & $-h$ & c & $\mathrm{f}$ & $-h$ & c & 0 & 0 & 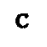 & 0 & 0 & \\
\hline 16 & & & & & & 17 & & & & & & 18 & & & & & \\
\hline a & 0 & $\mathbf{f}$ & a & 0 & f & $\mathbf{a}$ & 0 & & & 0 & & $\mathbf{a}$ & d & & & & \\
\hline 0 & $\mathbf{b}^{\prime}$ & 0 & 0 & $\mathbf{b}$ & 0 & 0 & $\mathrm{~b}$ & $\mathbf{h}$ & & b & $\mathbf{h}$ & e & b & 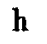 & 0 & 0 & \\
\hline$-\mathbf{f}$ & 0 & c & -f & 0 & c & 0 & $-h$ & c & 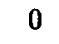 & $-h$ & 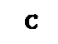 & $g$ & & c & 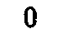 & 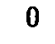 & \\
\hline 19 & & & & & & 20 & & & & & & 21 & & & & & \\
\hline 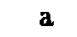 & 0 & 0 & 0 & $\mathrm{~d}$ & $f$ & 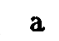 & 0 & & 0 & $\mathrm{~d}$ & 0 & $\mathbf{a}$ & d & & & & \\
\hline 0 & b & $\mathbf{h}$ & e & 0 & 0 & 0 & b & 0 & & & h & e & & 0 & & & \\
\hline 0 & $\mathbf{i}$ & $\mathbf{c}$ & $\mathbf{g}$ & 0 & 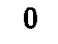 & & 0 & c & 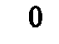 & & 0 & 0 & 0 & c & $g$ & $\mathbf{i}$ & \\
\hline 22 & & & & & & 23 & & & & & & 24 & & & & & \\
\hline $\mathbf{a}$ & 0 & 0 & 0 & 0 & 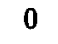 & $\mathbf{a}$ & 0 & & & & & $\mathbf{a}$ & 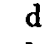 & & & & \\
\hline 0 & b & $\mathbf{h}$ & 0 & 0 & 0 & 0 & b & 0 & & & 0 & 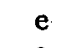 & b & 0 & 0 & & \\
\hline 0 & $\mathbf{i}$ & $\mathbf{c}$ & 0 & 0 & 0 & & 6 & 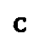 & 0 & 0 & 0 & & 0 & c & 0 & 0 & 0 \\
\hline 25 & & & & & & 26 & & & & & & 27 & & & & & \\
\hline 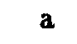 & 0 & 0 & 0 & 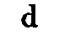 & 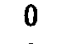 & 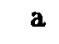 & 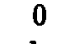 & & & & & 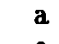 & 0 & & 0 & & \\
\hline 0 & b & 0 & & 0 & 0 & U & b & 0 & 0 & 0 & $\mathbf{h}$ & 0 & b & 0 & 0 & & \\
\hline & & & & & & & & & & & & & & & & & \\
\hline
\end{tabular}


TABLE III cont.

\begin{tabular}{|c|c|c|c|c|c|c|c|c|c|c|c|c|c|c|c|c|c|}
\hline 28 & & & & & & 29 & & & & & & $\overline{30}$ & & & & & \\
\hline $\mathbf{a}$ & 0 & 0 & 0 & 0 & 0 & $\mathbf{a}$ & d & 0 & 0 & 0 & 0 & $\mathbf{a}$ & 0 & 0 & 0 & d & 0 \\
\hline 0 & $\mathbf{b}$ & 0 & 0 & 0 & 0 & $-d$ & $\mathbf{a}$ & 0 & 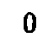 & 0 & 0 & 0 & $\mathbf{a}$ & 0 & $-d$ & 0 & 0 \\
\hline 0 & 0 & c & 0 & 0 & 0 & 0 & & 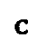 & 0 & 0 & 0 & 0 & 0 & c & 0 & 0 & 0 \\
\hline 31 & & & & & & 32 & & & & & & 33 & & & & & \\
\hline $\mathbf{a}$ & 0 & 0 & 0 & 0 & 0 & a & d & 0 & $\mathbf{a}$ & d & 0 & $\mathbf{a}$ & 0 & 0 & 0 & $\mathrm{~d}$ & 0 \\
\hline 0 & $\mathbf{a}$ & 0 & 0 & 0 & 0 & $-d$ & $\mathbf{a}$ & 0 & d & $-a$ & 0 & 0 & $\mathbf{a}$ & 0 & $\mathrm{~d}$ & 0 & 0 \\
\hline 0 & 0 & c & 0 & 0 & 0 & 0 & 0 & c & 0 & 0 & 0 & 0 & 0 & c & 0 & 0 & 0 \\
\hline 34 & & & & & & 35 & & & & & & 36 & & & & & \\
\hline $\mathbf{a}$ & 0 & 0 & $\mathbf{a}$ & $d$ & 0 & $\mathbf{a}$ & 0 & 0 & $\mathbf{a}$ & $d$ & 0 & $\mathbf{a}$ & 0 & 0 & 0 & $\mathrm{~d}$ & \\
\hline 0 & $\mathbf{a}$ & 0 & $d$ & $-\mathbf{a}$ & 0 & 0 & a & 0 & $\mathbf{e}$ & $-\mathbf{a}$ & 0 & 0 & $a$ & 0 & e & 0 & 0 \\
\hline 0 & 0 & c & 0 & 0 & 0 & 0 & 0 & $c$ & 0 & 0 & 0 & 0 & 0 & c & 0 & 0 & 0 \\
\hline 37 & & & & & & 38 & & & & & & 39 & & & & & \\
\hline $\mathbf{a}$ & $\mathrm{d}$ & 0 & 0 & $\mathrm{~d}$ & 0 & $\mathbf{a}$ & 0 & 0 & $\mathbf{a}$ & 0 & 0 & $\mathbf{a}$ & d & 0 & a & 0 & 0 \\
\hline$-d$ & $\mathbf{a}$ & 0 & $d$ & 0 & 0 & 0 & $\mathbf{a}$ & 0 & 0 & $-\mathbf{a}$ & 0 & $-d$ & $\mathbf{a}$ & 0 & 0 & $-\mathbf{a}$ & 0 \\
\hline 0 & 0 & c & 0 & 0 & 0 & 0 & 0 & $\mathbf{C}$ & 0 & 0 & 0 & 0 & & $c$ & 0 & 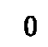 & 0 \\
\hline 40 & & & & & & 41 & & & & & & 42 & & & & & \\
\hline a & $d$ & $f$ & 0 & 0 & 0 & $\mathbf{a}$ & $\mathrm{d}$ & & 0 & $d$ & f & $\mathbf{a}$ & 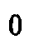 & $c$ & 0 & d & \\
\hline $\mathrm{d}$ & $\mathbf{b}$ & $\mathbf{h}$ & 0 & 0 & 0 & $d$ & b & 1 & $-d$ & 0 & $\mathbf{h}$ & 0 & b & $\mathbf{n}$ & d & $\mathrm{t}$ & 0 \\
\hline$f$ & $\mathbf{h}$ & c & 0 & 0 & 0 & f & $\mathbf{h}$ & $\mathbf{C}$ & $-f$ & $-h$ & 0 & 0 & $\mathbf{h}$ & & 1 & 0 & 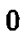 \\
\hline 43 & & & & & & 44 & & & & & & 45 & & & & & \\
\hline $\mathbf{a}$ & 0 & $f$ & 0 & $d$ & 0 & $\mathbf{a}$ & d & & 0 & 0 & 1 & $\mathbf{a}$ & d & 0 & & 0 & 0 \\
\hline 0 & $\mathbf{b}$ & 0 & $d$ & 0 & $\mathbf{h}$ & $\mathrm{d}$ & b & ( & 0 & 0 & $\mathrm{~h}$ & $\mathrm{~d}$ & b & & & & 0 \\
\hline$f$ & 0 & $c$ & 0 & $\mathbf{h}$ & 0 & 0 & U & $\mathbf{C}$ & $\mathbf{f}$ & $\mathbf{h}$ & 0 & 0 & 0 & C & 0 & 0 & 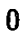 \\
\hline 46 & & & & & & 47 & & & & & & 48 & & & & & \\
\hline $\mathbf{a}$ & 0 & $\mathbf{f}$ & 0 & 0 & 0 & a & 0 & & 0 & 0 & 0 & $\mathbf{a}$ & 0 & & & & 0 \\
\hline 0 & $\mathbf{b}$ & 0 & 0 & 0 & 0 & 0 & b & $\mathbf{h}$ & 0 & 0 & 0 & 0 & h & & d & 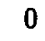 & 0 \\
\hline $\mathbf{f}$ & 0 & c & 0 & 0 & 0 & 0 & h & c & 0 & 0 & 0 & 0 & 0 & C & 0 & 0 & 0 \\
\hline 49 & & & & & & 50 & & & & & & 51 & & & & & \\
\hline a & 0 & 0 & 0 & 0 & 0 & $\mathbf{a}$ & 0 & & 0 & & & $\mathbf{a}$ & $\mathrm{d}$ & 1 & 0 & $d$ & $f$ \\
\hline 0 & $\mathbf{b}$ & 0 & 0 & 0 & $\mathbf{h}$ & 0 & $\mathbf{b}$ & & 0 & 0 & 0 & $-d$ & b & $h$ & d & 0 & h \\
\hline $\mathbf{0}$ & 0 & c & 0 & $\mathbf{h}$ & 0 & 0 & 0 & C & $\mathbf{f}$ & 0 & 0 & $-f$ & $\mathbf{h}$ & c & $f$ & $-h$ & 0 \\
\hline 52 & & & & & & 53 & & & & & & 54 & & & & & \\
\hline $\mathbf{a}$ & $\mathrm{d}$ & 1 & 0 & $\mathrm{~d}$ & 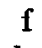 & $\mathbf{a}$ & d & 1 & 0 & d & + & $\mathbf{a}$ & 0 & 0 & 0 & $d$ & $\mathbf{f}$ \\
\hline$-d$ & $\mathbf{b}$ & $\mathbf{h}$ & $d$ & 0 & $\mathbf{h}$ & d & b & h & $-d$ & 0 & $\mathbf{h}$ & 0 & b & $\mathbf{h}$ & $-d$ & 0 & 0 \\
\hline$f$ & $-\mathbf{h}$ & c & $-f$ & $\mathbf{h}$ & 0 & $-\mathbf{f}$ & -h & $\mathbf{c}$ & f & $\mathbf{h}$ & 0 & 0 & $\mathbf{h}$ & $\mathbf{C}$ & $-f$ & 0 & 0 \\
\hline 55 & & & & & & 56 & & & & & & 57 & & & & & \\
\hline $\mathbf{a}$ & 0 & c & & $\mathrm{d}$ & 0 & 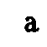 & & & & & 0 & $\mathbf{a}$ & & & & 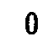 & 0 \\
\hline 0 & $\mathbf{b}$ & 0 & $-d$ & 0 & $\mathbf{i l}$ & d & b & 0 & $-d$ & 0 & 0 & 0 & b & $\mathbf{h}$ & 0 & 0 & $\mathbf{h}$ \\
\hline$f$ & 0 & c & 0 & $-h$ & 0 & 0 & 0 & & 0 & 0 & 0 & 0 & $\mathbf{h}$ & $\mathbf{c}$ & 0 & $-h$ & 0 \\
\hline
\end{tabular}


TABLE III cont.

\begin{tabular}{|c|c|c|c|c|c|c|c|c|c|c|c|c|c|c|c|c|c|}
\hline 58 & & & & & & 59 & & & & & & 60 & & & & & \\
\hline a & 0 & f & 0 & 0 & f & $\mathbf{a}$ & d & 0 & 0 & 0 & $\mathbf{f}$ & $a$ & $\mathrm{~d}$ & $\mathbf{f}$ & 0 & 0 & 0 \\
\hline 0 & b & 0 & 0 & 0 & 0 & $\mathrm{~d}$ & b & 0 & 0 & 0 & $\mathbf{h}$ & $-d$ & $\mathrm{~b}$ & $\mathbf{h}$ & 0 & 0 & 0 \\
\hline $\mathbf{f}$ & 0 & c & -f & 0 & 0 & 0 & 0 & c & $-f$ & $-\mathbf{h}$ & 0 & -1 & $\mathbf{h}$ & $\mathbf{c}$ & 0 & 0 & 0 \\
\hline 61 & & & & & & 62 & & & & & & 63 & & & & & \\
\hline $\mathbf{a}$ & d & f & 0 & 0 & 0 & $\mathbf{a}$ & $d$ & $\mathbf{f}$ & 0 & 0 & 0 & $\mathbf{a}$ & $\mathrm{d}$ & 0 & 0 & d & 0 \\
\hline$-d$ & $\mathrm{~b}$ & $\mathrm{~h}$ & 0 & 0 & 0 & $\mathrm{~d}$ & b & h & 0 & 0 & 0 & $-\mathrm{d}$ & $\mathrm{b}$ & 0 & d & 0 & 0 \\
\hline$f$ & $-\mathrm{h}$ & c & 0 & 0 & 0 & $-f$ & $-h$ & c & 0 & 0 & 0 & 0 & 0 & $\mathbf{c}$ & 0 & 0 & 0 \\
\hline 64 & & & & & & 65 & & & & & & 66 & & & & & \\
\hline a & 0 & f & 0 & d & 0 & $\mathbf{a}$ & 0 & 0 & 0 & d & f & $\mathbf{a}$ & 0 & f & 0 & d & 0 \\
\hline 0 & $\mathrm{~b}$ & 0 & d & 0 & $\mathbf{h}$ & 0 & b & $\mathbf{h}$ & $\mathrm{d}$ & 0 & 0 & 0 & b & 0 & $-d$ & 0 & $\mathbf{h}$ \\
\hline$-f$ & 0 & c & 0 & $-h$ & 0 & 0 & $-h$ & c & $-f$ & 0 & 0 & $-f$ & 0 & c & 0 & $\mathbf{h}$ & 0 \\
\hline 67 & & & & & & 68 & & & & & & 69 & & & & & \\
\hline $\mathbf{a}$ & 0 & 0 & 0 & d & f & $\mathbf{a}$ & d & 0 & 0 & 0 & f & $\mathbf{a}$ & $\mathrm{d}$ & 0 & 0 & 0 & f \\
\hline 0 & $\mathrm{~b}$ & h & $-d$ & 0 & 0 & $-d$ & b & 0 & 0 & 0 & $\mathbf{h}$ & $-\mathrm{d}$ & b & 0 & 0 & 0 & $\mathbf{h}$ \\
\hline 0 & $-\mathrm{h}$ & c & f & 0 & 0 & 0 & 0 & $c$ & $-f$ & $\mathbf{h}$ & 0 & 0 & 0 & $\mathbf{c}$ & f & $-h$ & 0 \\
\hline 70 & & & & & & 71 & & & & & & 72 & & & & & \\
\hline $\mathbf{a}$ & 0 & $\mathrm{f}$ & 0 & 0 & f & $\mathbf{a}$ & 0 & 0 & 0 & 0 & 0 & $\mathbf{a}$ & 0 & 0 & 0 & d & 0 \\
\hline 0 & $\mathrm{~b}$ & 0 & 0 & 0 & 0 & 0 & b & $\mathbf{h}$ & 0 & 0 & $\mathbf{h}$ & 0 & $\mathrm{~b}$ & 0 & $-d$ & 0 & 0 \\
\hline$-f$ & 0 & c & f & 0 & 0 & 0 & $-\mathrm{h}$ & c & 0 & $\mathbf{h}$ & 0 & 0 & 0 & c & 0 & 0 & 0 \\
\hline 73 & & & & & & 74 & & & & & & 75 & & & & & \\
\hline $\mathbf{a}$ & 0 & 0 & 0 & 0 & 0 & a & 0 & 0 & 0 & 0 & $f$ & $\mathbf{a}$ & $\mathrm{d}$ & 0 & 0 & 0 & 0 \\
\hline 0 & b & 0 & 0 & 0 & h & 0 & b & 0 & 0 & 0 & 0 & $-\mathrm{d}$ & $\mathrm{b}$ & 0 & 0 & 0 & 0 \\
\hline 0 & 0 & c & 0 & $-h$ & 0 & 0 & 0 & c & $-f$ & 0 & 0 & 0 & 0 & c & 0 & 0 & 0 \\
\hline 76 & & & & & & 77 & & & & & & 78 & & & & & \\
\hline $\mathbf{a}$ & 0 & f & 0 & 0 & 0 & $\mathbf{a}$ & 0 & 0 & 0 & 0 & 0 & $\mathbf{a}$ & $\mathrm{d}$ & f & & & \\
\hline 0 & b & 0 & 0 & 0 & 0 & 0 & $\mathbf{b}$ & h & 0 & 0 & 0 & $\mathbf{e}$ & $\mathbf{b}$ & $\mathbf{h}$ & & & \\
\hline$-f$ & 0 & c & 0 & 0 & 0 & 0 & $-h$ & c & 0 & 0 & 0 & g & $\mathbf{i}$ & $\mathbf{c}$ & & & \\
\hline 79 & & & & & & 80 & & & & & & 81 & & & & & \\
\hline $\mathbf{a}$ & d & 0 & & & & $\mathbf{a}$ & 0 & 0 & & & & a & $\mathrm{d}$ & 0 & & & \\
\hline e & b & 0 & & & & 0 & b & 0 & & & & $-d$ & $\mathbf{a}$ & 0 & & & \\
\hline 0 & 0 & c & & & & 0 & 0 & c & & & & 0 & 0 & $\mathbf{c}$ & & & \\
\hline 82 & & & & & & 83 & & & & & & 84 & & & & & \\
\hline $\mathbf{a}$ & 0 & 0 & & & & $\mathbf{a}$ & 0 & 0 & & & & $\mathbf{a}$ & d & $\mathbf{f}$ & & & \\
\hline 0 & $\mathbf{a}$ & 0 & & & & 0 & $\mathbf{a}$ & 0 & & & & $\mathrm{~d}$ & $b$ & $\mathbf{h}$ & & & \\
\hline 0 & 0 & c & & & & 0 & 0 & $\mathbf{a}$ & & & & $\mathrm{f}$ & $\mathbf{h}$ & c & & & \\
\hline
\end{tabular}


TABLE III cont.

\begin{tabular}{|c|c|c|c|c|c|c|c|c|}
\hline 85 & & & $\overline{86}$ & & & $\overline{87}$ & & \\
\hline $\mathbf{a}$ & d & f & & $\mathrm{d}$ & 0 & $\mathbf{a}$ & $\mathrm{d}$ & 0 \\
\hline $\mathrm{d}$ & b & $\mathbf{h}$ & & b & 0 & $-d$ & $\mathbf{b}$ & c \\
\hline$-\mathbf{f}$ & $-h$ & c & 0 & 0 & c & 0 & 0 & 0 \\
\hline 88 & & & & & & & & \\
\hline $\mathbf{a}$ & 0 & f & & & & & & \\
\hline 0 & b & 0 & & & & & & \\
\hline$-f$ & 0 & $c$ & & & & & & \\
\hline
\end{tabular}

\section{References}

[1] S. Malinowski, Acla Phys. Pol. A77, 555 (1990).

[2] S. Malinowski, Acla Phys. Pol. A77, 543 (1990).

[3] S. Malinowski, Acta Phys. Pol. A78, 921 (1990).

[4] R.M. White, Quantum Theory of Magnetism, Springer-Verlag, Berlin, Heidelberg, New York 1983.

[5] B.B. Krichevtsov, R.V. Pisarev, A.G. Selitsky, Sov. Phys.-Solid State 30, 2139 (1988).

[6] S. Bluck, H.G. Kahle, J. Phys. C, Solid State Phys. 21, 5193 (1988).

[7] R.V. Pisarev, B.B. Krichevtsov, V.V. Pavlov, A.G. Selitsky, J. Magn. Soc. Jpn. 11, Ns1, 33 (1987).

[8] B.B. Krichevtsov, V.V. Pavlov, R.V. Pisarev, Sov. Phys.-JETP Letl. 44, $471(1986)$.

[9] V.V. Bryksin, L.L. Korovin, M.P. Petrov, A.V. Chomenko, Sov. Phys.-Tech. Phys. 57, 1918 (1987).

[10] B.B. Krichevtsov, R.V. Pisarev, A.G. Selitsky, Sov. Phys.-JETP Lett. 41, 259 (1985).

[11] S.Yu. Davydov, E.I. Leonov, Sov. Phys.-Solid State 30, 1326 (1988).

[12] M. Aillerie, M.D. Fontana, F. Abdi, C. Carabatos-Nedelec, N. Thofanous, G. Alexakis, J. Appl. Phys. 65, 2406 (1988).

[13] II. Nakatani, W. Bosenberg, L.K. Cheng, C.L. Tang, Appl. Phys. Lett. 52,1288 (1988).

[14] J.F. Nye, Physical Properties of Crystals, Clarendon Press, Oxford 1957.

[15] R.R. Birss, Symmetry and Magnetism, North-Holland, Amsterdam 1964.

[16] R. Vlokh, Yu. Pyatak, I.P. Skab, Sov. Phys.-Cryslallogr. 34, 498 (1989).

[17] B.L. Greene, M. Thakur, J. Orenstein, Appl. Phys. Lett. 54, 2065 (1989). 